\title{
COMPACT FOURIER ANALYSIS FOR DESIGNING MULTIGRID METHODS
}

\author{
THOMAS K. HUCKLE*
}

\begin{abstract}
The convergence of Multigrid methods can be analyzed based on a Fourier analysis of the method or by proving certain inequalities that have to be fulfilled by the smoother and by the coarse grid correction separately. Here, we analyze the Multigrid method for the constant coefficient Poisson equation with a compact Fourier analysis using the formalism of multilevel Toeplitz matrices and their generating functions or symbols. The Fourier analysis is applied for determining the smoothing factor and the overall error of the combined smoothing and coarse grid correction error reduction of a Twogrid step by representing the Twogrid step explicitly by a symbol. If the effects of the smoothing correction and the coarse grid correction are orthogonal to each other, then in a Twogrid step the error is removed in one step and the Twogrid method can be considered as a direct solver. If the coarse linear system is identical to the original matrix, then the same projection and smoother again make the Twogrid step a direct solver. Hence, the multigrid cycle has to be applied only once with one smoothing step on each level and therefore the whole Multigrid method can be considered as a direct solver. In this paper we want to identify Multigrid as a direct solver in 1D with coarse system derived by linear and constant interpolation, and in 2D for a modified projection related to [21, paragraph A.2.3]. By studying not only smoothing and coarse grid correction separately but the symbol of the full Twogrid step as well, we are furthermore able to derive better convergence estimates and information on how to find efficient combinations of projection and smoother. As smoothers we consider also approximate inverse smoothers, colored smoothers that take into account the different character of grid points, and rank reducing smoothers that coincide with the given matrix on a large number of entries. Numerical examples show the effectiveness of the new approach.
\end{abstract}

Key words. Multigrid, Toeplitz, Block Toeplitz, Fourier Analysis, generating function

AMS subject classifications. $65 \mathrm{~F} 10,65 \mathrm{~F} 15$

1. Introduction. We consider discretizations of the constant coefficient Poisson equation, e.g. in two dimensions

$$
u_{x x}+u_{y y}=f(x, y)
$$

with Dirichlet boundary conditions that lead to a linear system $A x=b$. As solver we apply the Multigrid method with standard coarsening defined by

1. the prolongation matrix $P$, which according to the Galerkin approach leads to the coarse grid matrix $A_{c}=P^{T} A P$,

2. and the smoother given by the matrix $M$, which defines the smoothing iteration $x_{k+1}=x_{k}+M^{-1}\left(b-A x_{k}\right)$.

In a first stage the last approximation is improved by applying a few steps of the smoothing iteration. Then the residual is smooth and can be represented on a coarser grid. The transformation from fine to coarse vectors is given through the restriction $P^{T}$. The solution on the coarse grid can be transformed back on the fine grid by the prolongation $P$. This describes the Twogrid method; a recursive formulation of this method - repeating the smoothing and the coarsening also on $A_{c}$ - leads to the Multigrid method.

The convergence of the Multigrid method can be analyzed via Fourier analysis making use of the intimate connection between the Laplacian with periodic boundary values and the discrete Fourier Transform, resp. trigonometric basis functions ([2, 21, $23,5])$. A second approach uses inequalities for the reduction of the error by coarse grid correction CGC and by the smoothing iteration to prove the convergence ([17]).

*Fakultät für Informatik, Technische Universität München, Boltzmannstr. 3, D-85748 Garching, Germany (huckle@in.tum.de). 
The matrices that have to be considered are

$$
C G C=I-P A_{c}^{-1} P^{T} A
$$

and the post- and presmoothing $S_{l}$ and $S_{r}$

$$
S_{l}=I-M_{l}^{-1} A, \quad S_{r}=I-M_{r}^{-1} A .
$$

The smoother can be given as an approximation $M$ of the matrix $A$ - like the GaussSeidel triangular factor - or as an approximation $N$ of the inverse $A^{-1}$ like a sparse approximate inverse; in this case $M^{-1}$ has to be replaced by $N$ in $S_{l}$ and $S_{r}$. The full error reduction of a Twogrid step TGS with pre- and postsmoothing is described by the matrix

(1.3) $T G S=S_{l} \cdot C G C \cdot S_{r}=\left(I-M_{l}^{-1} A\right) \cdot\left(I-P A_{c}^{-1} P^{T} A\right) \cdot\left(I-M_{r}^{-1} A\right)$.

The standard convergence analysis is based on analyzing $C G C, S_{l}$ and $S_{r}$. In this paper we are interested in Multigrid as a direct solver ([3] and [21, paragraph A.2.3]. This is equivalent to the property that $T G S=0$ and the coarse matrix $A_{c}$ differs from $A$ only in the dimension, but has the same coefficients except for a constant factor. So the task is to identify a pair of projection $P$ and smoother $M$, resp. $N$, that leads to $T G S=0$. If this cannot be achieved then at least we can try to adjust the projection and the smoother in such a way that the TGS-symbol is of small rank and norm. The tool we are using in the following is a compact Fourier analysis developed for Multigrid methods for Toeplitz matrices ([7, 8, 4, 1, 20, 13, 14, 9, 10]). In contrast to the classical Fourier analysis, here we consider the constant coefficient case with Dirichlet boundary conditions resulting in the 1D case in a Toeplitz matrix

$$
T_{n}=T_{n}(f)=\left(\begin{array}{ccccc}
t_{0} & t_{-1} & \cdots & \cdots & t_{1-n} \\
t_{1} & t_{0} & t_{-1} & & \vdots \\
\vdots & \ddots & \ddots & \ddots & \vdots \\
\vdots & & \ddots & \ddots & t_{-1} \\
t_{n-1} & \cdots & \cdots & t_{1} & t_{0}
\end{array}\right) .
$$

The analysis is then based on the symbol or generating function of $T_{n}$ :

$$
f(x)=\sum_{j=-\infty}^{\infty} t_{j} e^{i j x} .
$$

For nonnegative $f \in L_{\infty}$, the full matrices and the symbol are connected in the way that the range of values (and therefore the eigenvalues) of the full matrices is contained in the range of values of the symbol ([11]). In order to analyze Multigrid methods we have to consider the given matrices as Block Toeplitz matrices in order to capture the two different classes of coefficients, the fine and the coarse grid unknowns. Also for Block Toeplitz matrices the relation between the full matrices and the symbol is described by the range of values $([19,12])$. Furthermore, also the range of values of $T_{n}(f) \cdot T_{n}^{-1}(g)$ is described by the the range of values $f(x) g^{-1}(x)$ for symmetric positive definite (spd) matrices ([18]), also in the block case. For higher dimensional PDEs we have to deal with multilevel Block Toeplitz matrices.

By transferring all Multigrid matrices (the given problem, the projection, the smoother, the coarse problem) into block symbols we can analyze the smoothing factor and the Twogrid error operators TGS in terms of small matrix functions. This can be used for finding combinations of discretization, projection, and smoother that lead 
to practical implementations of Multigrid as a direct solver or - if this is not possible - we can analyze the related block symbols in order to reduce the rank or the norm of the TGS symbol to ensure fast convergence. This representation by block symbols is especially helpful for analyzing colored smoothers, and has been applied already for deriving Multigrid methods with general constant coefficients like the Helmholtz equation and anisotropic problems $([9,10])$. Furthermore, in a forthcoming paper this approach will be used to analyze aggregation-based Multigrid methods ([16]) where the choice of the smoother is crucial to overcome the disadvantages introduced by applying the somewhat crude aggregation technique. This analysis can be also helpful for defining practical projections and smoothers for PDEs with varying coefficients similar to the Local Fourier Analysis (LFA) [2, 21, 23].

In Section 2 we develop the compact Fourier analysis for the 1D Laplacian case by considering all involved matrices as Toeplitz, resp. Block Toeplitz matrices and their underlying symbols. By considering the symbol of the $T G S$-matrix we are able to define prolongations and smoothers that lead to a direct solver for different problems in 1D. Especially we find efficient Multigrid forms for MG with constant interpolation.

In Section 3 we generalize the results to the more interesting 2D case by determining a combination of projection and postsmoother that leads to a Twogrid direct solver. We also identify general conditions for smoothers that yield Multigrid as a direct solver and generalize the results from [21, paragraph A.2.3]. Furthermore, we analyze different smoothers for the 5- and the 9-point stencil. Considering the combination of smoother and projection in the $T G S$-symbol shows that the quality of a smoother can strongly depend on the way the smoother and its transpose are applied. Furthermore, the advantages of approximate inverse smoothers and rank reducing smoothers are displayed. In the final conclusions in Section 4 we discuss the results of the new technique.

2. Compact Fourier analysis in the $1 \mathrm{D}$ case. In a first step we have to represent all matrices by block symbols. The given linear system with three point stencil is $A x=b$ with

$$
A=\left(\begin{array}{cccc}
2 & -1 & & \\
-1 & 2 & \ddots & \\
& \ddots & \ddots & -1 \\
& & -1 & 2
\end{array}\right)=T_{n}(f)
$$

Then $A$ is related to the scalar symbol $f(x)=-1 \cdot \exp (i x)+2-1 \cdot \exp (-i x)=$ $2(1-\cos (x))$. We can obtain the block symbol $F(x)$ in two different ways. In a first attempt we partition $A$ in $2 \times 2$ blocks in the form

$$
A=\left(\begin{array}{cc|cc|cc|c}
2 & -1 & 0 & 0 & 0 & 0 & \cdot \\
-1 & 2 & -1 & 0 & 0 & 0 & \cdot \\
\hline 0 & -1 & 2 & -1 & 0 & 0 & \cdot \\
0 & 0 & -1 & 2 & -1 & 0 & . \\
\hline \cdot & . & . & . & . & . & .
\end{array}\right)=T_{n}(F)
$$

Hence, the block symbol $F(x)=\sum_{j=-\infty}^{\infty} T_{j} e^{i j x}$ has the form

$$
F(x)=\left(\begin{array}{cc}
0 & -1 \\
0 & 0
\end{array}\right) \exp (i x)+\left(\begin{array}{cc}
2 & -1 \\
-1 & 2
\end{array}\right)+\left(\begin{array}{cc}
0 & 0 \\
-1 & 0
\end{array}\right) \exp (-i x)
$$




$$
=\left(\begin{array}{cc}
2 & -1-\exp (i x) \\
-1-\exp (-i x) & 2
\end{array}\right)=\left(\begin{array}{cc}
2 & -\alpha \\
-\bar{\alpha} & 2
\end{array}\right)
$$

with $\alpha=1+\exp (i x)$. Then, index 1 in $F$ is representing fine grid points that are also coarse, while index 2 is related to grid points that appear only in the fine grid. Similarly, the odd row or column indices in each $2 \times 2$ block of the matrix $A$ are related to coarse points and the even to noncoarse points.

A more formal approach derives the coefficients of the block matrix function $F(x)$ directly from the coefficients of the scalar function $f(x)$. We observe, that every second coefficient in the blockwise form of $A$ is related to every second entry in the series expansion of $f(x)$, and therefore the coefficients in the block symbol are given by $(f(x / 2)+f(x / 2+\pi)) / 2$ or $\exp ( \pm i x / 2) \cdot(f(x / 2)-f(x / 2+\pi)) / 2$. So, the block symbol to a scalar function $f(x)$ has the form

$$
F(x)=\left(\begin{array}{cc}
\frac{f(x / 2)+f(x / 2+\pi)}{2} & \exp \left(\frac{i x}{2}\right) \cdot \frac{f(x / 2)-f(x / 2+\pi)}{2} \\
\exp \left(\frac{-i x}{2}\right) \cdot \frac{f(x / 2)-f(x / 2+\pi)}{2} & \frac{f(x / 2)+f(x / 2+\pi)}{2}
\end{array}\right) .
$$

The full weighting restriction from fine to coarse grid is given by

$$
R=\left(\begin{array}{cccccccc}
1 & 2 & 1 & & & & \\
& & 1 & 2 & 1 & & \\
& & & & 1 & 2 & 1 & \\
& & & & & . & . & .
\end{array}\right)=P^{T}
$$

which can be seen as picking every second row out of the $n \times n$ Toeplitz matrix $B=\operatorname{tridiag}(1,2,1)=B^{T}=B^{H}$, or with the trivial injection

$$
E=\left(\begin{array}{cccccc}
1 & 0 & & & & \\
& 0 & 1 & 0 & & \\
& & & 0 & 1 & \\
& & & & . & .
\end{array}\right)
$$

we have $R=E \cdot B$ and the Galerkin coarse system

$$
A_{c}=P^{T} A P=R A R^{T}=E\left(B A B^{T}\right) E^{T} .
$$

Hence, the standard projection by linear interpolation is related to the matrix $\operatorname{tridiag}(1,2,1) / 2$ or the scalar symbol $b(x)=1+\cos (x)$ with block symbol

$$
B(x)=\left(\begin{array}{cc}
1 & (1+\exp (i x)) / 2 \\
(1+\exp (-i x)) / 2 & 1
\end{array}\right)=\left(\begin{array}{cc}
1 & \alpha / 2 \\
\bar{\alpha} / 2 & 1
\end{array}\right) .
$$

Coarsening is equivalent to picking only the first column/row in the block symbol. Therefore, the scalar symbol for the coarse problem related to $A_{c}$ is given by

$$
\begin{gathered}
\left(\begin{array}{cc}
1 & 0
\end{array}\right) B(x) \cdot F(x) \cdot B(x)\left(\begin{array}{l}
1 \\
0
\end{array}\right)=B_{1} F B_{1}^{H}=\left(\begin{array}{ll}
1 & \alpha / 2
\end{array}\right) \cdot\left(\begin{array}{cc}
2 & -\alpha \\
-\bar{\alpha} & 2
\end{array}\right) \cdot\left(\begin{array}{c}
1 \\
\bar{\alpha} / 2
\end{array}\right) \\
=2\left(1-|\alpha|^{2} / 4\right)=1-\cos (x)=f(x) / 2=: f_{c}(x)
\end{gathered}
$$

with $B_{1}=B(1,:)$ the vector of the first row of the symbol $B$.

This reflects an important property of Multigrid methods, namely that the coarse problem is similar to the original matrix in the sense that the symbols share at least the same singularity $x_{0}=0$. For the Laplacian the symbol $f(x)$ of the given matrix 
has a zero of order two (at $x_{0}=0$ ). Following [7], the prolongation has to be related to a symbol $b(x)$ with zero at the mirror point $\left(x_{0}+\pi\right)$ in order to derive an appropriate coarse grid symbol.

Next we restrict ourselves to the case where the smoother $M$ (or its inverse $N$ ) is also a Block Toeplitz matrix, represented e.g. by

$$
M(x)=\left(\begin{array}{ll}
M_{1,1}(x) & M_{1,2}(x) \\
M_{2,1}(x) & M_{2,2}(x)
\end{array}\right) \quad \text { or } N(x)=\left(\begin{array}{ll}
N_{1,1}(x) & N_{1,2}(x) \\
N_{2,1}(x) & N_{2,2}(x)
\end{array}\right) .
$$

For explicit smoothers $M$ we have to keep in mind that in each smoothing iteration we have to solve a linear system with $M$. Therefore, $M$ should be a matrix that is easy to invert, e.g. $M$ or $M(x)$ should be upper or lower triangular, or even diagonal. For an approximate inverse smoother $N$ we do not need the inverse, $N$ is even allowed to be singular. We have only to be careful that the entries in $N$ should be given by polynomials in $\exp ( \pm i x)$ in order to obtain sparsity. Because of this different character of explicit smoother $M$ or approximate inverse smoother $N$ we emphasize this distinction in the following.

Then, from equations (1.1), (1.2), and (1.3) the full Twogrid step can be described by the $2 \times 2$-symbol

$$
T G S(x)=\left(I-M_{l}^{-1}(x) F(x)\right)\left(I-\frac{B_{1}^{H}(x) B_{1}(x) F(x)}{f_{c}(x)}\right)\left(I-M_{r}^{-1}(x) F(x)\right),
$$

or with $M^{-1}$ replaced by $N$ for the case of approximate inverse smoother.

A direct solver can only be derived if one of the $2 \times 2$ matrices TGS from (2.5) with smoothers $M$ or $N$ is zero (see Theorem 2.1 below). Note, that in view of the Galerkin approach the $2 \times 2$ symbol for $C G C$ is of rank 1 , because it holds

$$
C G C(x) \cdot B_{1}^{H}=B_{1}^{H}-B_{1}^{H}\left(B_{1} F(x) B_{1}^{H}\right) / f_{c}(x) \equiv 0 .
$$

Hence, to obtain $T G S(x) \equiv 0$ the smoother has only to take care of the remaining one-dimensional subspace. If the coarse grid system is replaced by a matrix different from the Galerkin projection then $T G S(x) \equiv 0$ can only be obtained by $M=A$, resp. $N=A^{-1}$, as smoother. This is due to a variational principle for the Galerkin operator, see e.g. [21, paragraph A.2.4].

The matrices in $T G S$ are sparse and banded, and can be described by block symbols. Let us collect some useful properties of such matrices $([22,18,19,12])$ (in the following $R_{j}$ denote matrices of small rank, independent of $n$ ):

1. For scalar banded Toeplitz matrices with symbols $f$ and $g$ trigonometric polynomials it holds

$$
\begin{aligned}
T_{n}(f) T_{n}(g)+R_{1} & =T_{n}(f g)=T_{n}(g f)=T_{n}(g) T_{n}(f)+R_{2} \\
T_{n}(g) T_{n}^{-1}(f) & =T_{n}^{-1}(f) T_{n}(g)+R_{3} .
\end{aligned}
$$

2. These results can be generalized to block matrices $F$ and $G$ with coefficients $F_{i j}$ and $G_{i j}$ trigonometric polynomials in the form

$$
T_{n}(F) T_{n}(G)=T_{n}(F G)+R_{4} .
$$

Note, that because of $F G \neq G F$ in general $T_{n}(F) T_{n}(G)=T_{n}(G) T_{n}(F)+R_{5}$ does not hold. But, if one of the block symbols is a scalar symbol $G(x)=g(x) \cdot I$, then we obtain

$$
T_{n}^{-1}(g I) T_{n}(F)=T_{n}(F) T_{n}^{-1}(g I)+R_{6}
$$

in the same way as in the scalar case. 
3. Let $M(x)$ and $F(x)$ be again trigonometric block symbols. The inverse of $M(x)$ can be written as $M^{-1}(x)=\operatorname{det}(M(x))^{-1} M_{+}(x)=d_{M}(x)^{-1} M_{+}(x)$ with the adjoint matrix $M_{+}$being again a trigonometric polynomial. Then it holds

$$
T_{n}\left(d_{M}\right)=T_{n}\left(M(x) M^{-1}(x) \cdot d_{M}(x)\right)=T_{n}\left(M M_{+}\right)=T_{n}(M) T_{n}\left(M_{+}\right)+R_{7} .
$$

Therefore,

$$
\begin{aligned}
I_{n} & =T_{n}(M) T_{n}\left(M_{+}\right) T_{n}^{-1}\left(d_{M}\right)+R_{8} \Longrightarrow T_{n}^{-1}(M)=T_{n}\left(M_{+}\right) T_{n}^{-1}\left(d_{M}\right)+R_{9} \\
& \Longrightarrow T_{n}^{-1}(M) T_{n}(F)=T_{n}\left(M_{+} F\right) T_{n}^{-1}\left(d_{M}\right)+R_{10}
\end{aligned}
$$

THEOREM 2.1. The Twogrid error reduction $T G S_{n}$ for the original $n \times n$ problem and the symbol for TGS $(x)$ are connected in the way that if the symbol TGS $(x) \equiv 0$ then also $T G S_{n}$ is zero up to a low rank term.

Proof. First we consider the case of approximate inverse smoothers $N$ with

$T G S_{n}=\left(I-T_{n}\left(N_{l}\right) T_{n}(F)\right) \cdot\left(I-T_{n}\left(B_{0}^{H}\right) T_{n}^{-1}\left(f_{c} I\right) T_{n}\left(B_{0}\right) T_{n}(F)\right) \cdot\left(I-T_{n}\left(N_{r}\right) T_{n}(F)\right)$ where $B_{0}$ has first row $B_{1}=B(1,:)$ extended by zeros to a $2 \times 2$ symbol. In view of the previous listed properties of Block Toeplitz matrices it holds

$$
\begin{aligned}
T G S_{n} & =T_{n}^{-1}\left(f_{c} I\right) \cdot T_{n}\left(I-N_{l} F\right) \cdot T_{n}\left(f_{c} I-B_{0}^{H} B_{0} F\right) \cdot T_{n}\left(I-N_{r} F\right)+R_{1}= \\
& =T_{n}^{-1}\left(f_{c} I\right) \cdot T_{n}\left(\left(I-N_{l} F\right)\left(f_{c} I-B_{0}^{H} B_{0} F\right)\left(I-N_{r} F\right)\right)+R_{2}= \\
& =T_{n}^{-1}\left(f_{c} I\right) \cdot T_{n}\left(\left(I-N_{l} F\right)\left(I-B_{0}^{H} B_{0} F / f_{c}\right)\left(I-N_{r} F\right) \cdot f_{c}\right)+R_{3} .
\end{aligned}
$$

Therefore, if the symbol $T G S(x)$ is zero then also $T G S_{n}$ is zero except for a low rank term. Next we consider smoothers $M_{l}$ and $M_{r}$ :

$T G S_{n}=\left(I-T_{n}^{-1}\left(M_{l}\right) T_{n}(F)\right)\left(I-T_{n}\left(B_{0}^{H}\right) T_{n}^{-1}\left(f_{c} I\right) T_{n}\left(B_{0}\right) T_{n}(F)\right)\left(I-T_{n}^{-1}\left(M_{r}\right) T_{n}(F)\right)$.

Like above by using especially the result 3 . of the above listed properties, we obtain

$$
\begin{aligned}
& T G S_{n}=T_{n}^{-1}\left(M_{l}\right) T_{n}^{-1}\left(f_{c} I\right) T_{n}\left(M_{l}-F\right) T_{n}\left(f_{c} I-B_{0}^{H} B_{0} F\right) T_{n}\left(d_{M} I-M_{+} F\right) T_{n}^{-1}\left(d_{M}\right)+R_{4} \\
& \quad=T_{n}^{-1}\left(M_{l}\right) T_{n}^{-1}\left(f_{c} I\right) T_{n}\left(\left(M_{l}-F\right)\left(f_{c} I-B_{0}^{H} B_{0} F\right)\left(d_{M} I-M_{+} F\right)\right) T_{n}^{-1}\left(d_{M}\right)+R_{5}= \\
& T_{n}^{-1}\left(M_{l}\right) T_{n}^{-1}\left(f_{c} I\right) T_{n}\left(M_{l}\left(I-M_{l}^{-1} F\right)\left(I-B_{0}^{H} B_{0} F / f_{c}\right)\left(I-M^{-1} F\right) d_{M} f_{c}\right) T_{n}^{-1}\left(d_{M}\right)+R_{6} .
\end{aligned}
$$

Therefore, again for the symbol satisfying $T G S(x) \equiv 0$, also the $n \times n$ matrix $T G S_{n}$ will be zero up to a low rank term.

Note, that a similar proof can be given for the $2 \mathrm{D}$ case, but then in view of the block structure of the $n^{2} \times n^{2}$ matrices, the low rank can be in the size of $n$.

Remark 1: For analyzing the smoothing behavior based on the block symbol we have to develop a new technique similar to the red-black local Fourier analysis $([21,23,5])$. For the scalar symbols $f(x)$ of $A$ and $m(x)$ of the smoother, one considers $1-m(x)^{-1} f(x)$ for $x \in[\pi / 2, \pi]$ representing the high frequency subspace. In the block case we have to analyze the matrix function $I-M^{-1}(x) F(x)$ for the high frequency components. In this case, the high frequency components are not described by the values of the block symbol for $x \in[\pi / 2, \pi]$. Instead, we have to identify the eigenvectors relative to nonsingular eigenvalues of $F(x)$ and consider $I-M^{-1}(x) F(x)$ restricted to the subspace relative to these eigenvectors. In the standard $1 \mathrm{D}$ case the eigenvectors of $F$ are described by the diagonalization

$$
F(x)=\left(\begin{array}{cc}
2 & -\alpha \\
-\bar{\alpha} & 2
\end{array}\right)=\left(\begin{array}{cc}
1 & 1 \\
\bar{\alpha} /|\alpha| & -\bar{\alpha} /|\alpha|
\end{array}\right)\left(\begin{array}{cc}
2-|\alpha| & 0 \\
0 & 2+|\alpha|
\end{array}\right)\left(\begin{array}{cc}
1 & \alpha /|\alpha| \\
1 & -\alpha /|\alpha|
\end{array}\right) .
$$


To see this, in a first step we transform $F(x)$ by a unitary diagonal equivalence transformation $\operatorname{diag}(1, \alpha /|\alpha|)$ into a real circulant matrix ([6]). The eigenvectors of the circulant matrix are then given by the $2 \times 2$ Fourier matrix $F_{2}$ related to the discrete Fourier transform. The eigenvalues of $F(x)$ can also be described in terms of the scalar symbol by $\lambda_{0}(x)=f(x / 2)=2(1-|\alpha| / 2)$ and $\lambda_{1}(x)=f(x / 2+\pi)=2(1+|\alpha| / 2)$. So we see that one of the eigenvalues is related to the high frequency part and the other to the low frequency part. Therefore, the subspace important to smoothing is given by the eigenvector corresponding to eigenvalue $2+|\alpha|=f(x / 2+\pi)$. Reducing a block symbol to the eigenspace corresponding to $\lambda_{1}$ is equivalent to reducing the related scalar symbol to the interval $[\pi / 2, \pi]$. In order to analyze the damped Jacobi smoother in the block symbol we have to reduce the block symbol to the appropriate eigenvector subspace via

$$
\frac{1}{2}\left(\begin{array}{ll}
1 & -\bar{\alpha} /|\alpha|
\end{array}\right)\left(I-\frac{\omega}{2}\left(\begin{array}{cc}
2 & -\alpha \\
-\bar{\alpha} & 2
\end{array}\right)\right)\left(\begin{array}{c}
1 \\
-\alpha /|\alpha|
\end{array}\right)=1-\omega(1+|\alpha| / 2),
$$

which leads for the extreme cases $|\alpha|=0$ and 2 to the optimal solution $\omega=2 / 3$ as in the scalar standard smoothing analysis.

Next, we want to describe the symbols for the standard smoothers. Vice versa we can derive smoothers in terms of their block symbol and describe their full matrix representation. The Gauss-Seidel smoother $\operatorname{tril}(A)$ is a lower triangular matrix given by the non-triangular block symbol

$$
L_{G S}(x)=\left(\begin{array}{cc}
2 & -e^{i x} \\
-1 & 2
\end{array}\right)
$$

Note, that the entries in the lower triangular part of $A$ are related to real entries in the lower triangular part of the symbol, or to entries in the block symbol that are real factors of terms $\exp (i j x)$.

On the other side, the lower triangular part of the symbol $F$ is given by

$$
L_{\tilde{G S}}(x)=\left(\begin{array}{cc}
2 & 0 \\
-\bar{\alpha} & 2
\end{array}\right) \longrightarrow L_{R B}=\left(\begin{array}{cc}
2 I & 0 \\
-T(\bar{\alpha}) & 2 I
\end{array}\right)
$$

with $L_{R B}$ the related full matrix after odd-even permutation. The full modified Gauss-Seidel smoother in original ordering has the form

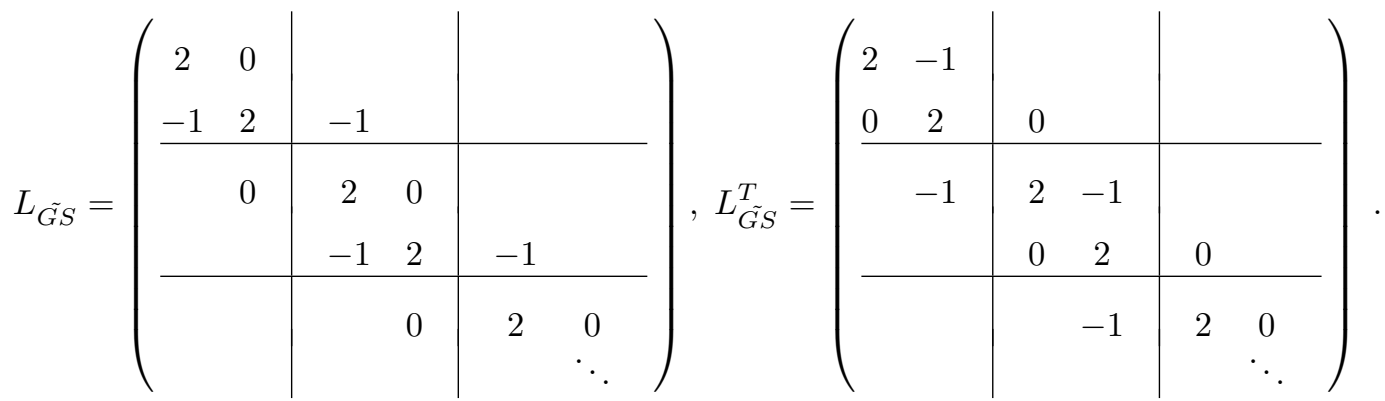

These two different matrices $L_{R B}$ and $L_{\tilde{G S}}$ are related to the two different interpretations of the block symbol. If the symbol $L_{\tilde{G S}}(x)$ is considered as representation of a Block Toeplitz matrix with small $2 \times 2$ blocks, the resulting full matrix is given by $L_{\tilde{G S}}$ and can be derived by deleting certain entries in the original matrix $A$. If $L_{\widetilde{G S}}(x)$ is considered as representation of a $2 \times 2$ Block Toeplitz matrix with large blocks, the resulting full matrix is $L_{R B}$. The transformation between these two interpretations 
of the matrices represented by the symbol is given by the the red-black (odd-even) reordering.

Because of the triangular form of the symbol $L_{\tilde{G S}}(x)$, in the full matrix $A$ every second column is replaced by the diagonal entry only. The lower triangular form $L_{\tilde{G S}}$ starts with replacing the second column, the upper triangular form $L_{\widetilde{G S}}^{T}$ starts with replacing the first column by the diagonal entry only. Hence, both smoothers treat the grid points in a different way according to their color. Obviously, linear equations in $L_{G S}$ and in $L_{R B}$ can be solved easily, also in parallel.

2.1. Multigrid as a direct solver for linear interpolation. The standard linear interpolation in 1D is given by the block symbol (2.4) and therefore the block symbol for $C G C$ in (1.1) is

$$
C G C(x)=I_{2}-B_{1}^{H} B_{1} F(x) / f_{c}(x)=\left(\begin{array}{cc}
0 & 0 \\
-\bar{\alpha} / 2 & 1
\end{array}\right) .
$$

For a direct solver we have to find post/pre-smoothers with $I_{2}-M(x) F(x)$ of rank 1 and

$$
\left(I_{2}-M_{l}^{-1}(x) F(x)\right) \cdot\left(\begin{array}{cc}
0 & 0 \\
-\bar{\alpha} / 2 & 1
\end{array}\right) \cdot\left(I_{2}-M_{r}^{-1}(x) \cdot F(x)\right) \equiv 0
$$

or the similar formula with $M^{-1}$ replaced by $N$ for approximate inverse smoothers. For the left postsmoother $N_{l}$ this yields the equations

$$
\left(\begin{array}{cc}
0 & 0 \\
-\bar{\alpha} / 2 & 1
\end{array}\right)=N_{l} \cdot\left(\begin{array}{cc}
2 & -\alpha \\
-\bar{\alpha} & 2
\end{array}\right)\left(\begin{array}{cc}
0 & 0 \\
-\bar{\alpha} / 2 & 1
\end{array}\right)=\left(\begin{array}{cc}
N_{1,1} & N_{1,2} \\
N_{2,1} & N_{2,2}
\end{array}\right)\left(\begin{array}{cc}
|\alpha|^{2} / 2 & -\alpha \\
-\bar{\alpha} & 2
\end{array}\right) .
$$

These equations lead to the conditions $N_{1,2}(x)=\alpha N_{1,1}(x) / 2$ and $N_{2,2}(x)=$ $0.5+\alpha N_{2,1}(x) / 2$ with the special solution $N_{l}=\left(\begin{array}{cc}0 & 0 \\ 0 & 0.5\end{array}\right)$.

For the smoother $M_{l}$ we get

$$
\left(\begin{array}{cc}
0 & 0 \\
-\bar{\alpha} / 2 & 1
\end{array}\right)=M_{l}^{-1} \cdot\left(\begin{array}{cc}
2 & -\alpha \\
-\bar{\alpha} & 2
\end{array}\right)\left(\begin{array}{cc}
0 & 0 \\
-\bar{\alpha} / 2 & 1
\end{array}\right)
$$

and therefore $M_{l}$ has to satisfy the condition

$$
M_{l} \cdot\left(\begin{array}{cc}
0 & 0 \\
-\bar{\alpha} / 2 & 1
\end{array}\right)=2\left(\begin{array}{cc}
|\alpha|^{2} / 4 & -\alpha / 2 \\
-\bar{\alpha} / 2 & 1
\end{array}\right)
$$

The general solution is given by $M_{l}(x)=\left(\begin{array}{cc}M_{1,1} & -\alpha \\ M_{2,1} & 2\end{array}\right)$ for any $M_{1,1}$ and $M_{2,1}$. In order to obtain a matrix that is easy to invert we can set $M_{l}=\left(\begin{array}{cc}2 & -\alpha \\ 0 & 2\end{array}\right)$, the upper triangular red-black Gauss-Seidel smoother.

In the same way we get for the presmoother on the right hand side the solutions $N_{2,1}=\bar{\alpha} N_{1,1} / 2$ and $N_{2,2}=0.5-\bar{\alpha} N_{1,2} / 2$, especially $N_{r}=\left(\begin{array}{cc}0 & 0 \\ 0 & 0.5\end{array}\right)$. Similarly, for $M_{r}$ we derive $M_{r}=\left(\begin{array}{cc}* & * \\ -\bar{\alpha} & 2\end{array}\right)$ with the special solution $M_{r}=\left(\begin{array}{cc}2 & 0 \\ -\bar{\alpha} & 2\end{array}\right)$.

Testing these smoothers for the full $n \times n$-matrix yields also $T G S_{n}=0$. Hence, not only the red-black Gauss-Seidel smoother gives MG as a direct solver, but also the approximate inverse smoother $N_{l}=N_{r}$ leads to a cheaper and more efficient solver. Especially, the smoother $N$ needs no solving of any e.g. triangular system, and changes only the values of the non-coarse entries with even indices. 
For the linear interpolation the CGC-symbol is of the special form with zeros in the first row. This case has been already described in [21, paragraph A.2.3] as impractical direct solver. In Section 3.2 we will analyze the matrix formulation of A.2.3 in terms of the block symbol to derive a more practical formulation.

2.2. Multigrid as direct solver for the constant interpolation. Next we consider the case, where we replace the standard projection with stencil $(1,2,1)$ by the constant projection $(1,1)$, related to the symbol $b(x)=1+\exp (i x)$. Then, the projection is given by $B_{1}(x)=(1,1)$, the first row of the block symbol $B(x)$, and the Galerkin coarse grid system has the symbol

$$
\left(\begin{array}{ll}
1 & 1
\end{array}\right)\left(\begin{array}{cc}
2 & -\alpha \\
-\bar{\alpha} & 2
\end{array}\right)\left(\begin{array}{l}
1 \\
1
\end{array}\right)=2-\exp (i x)-\exp (-i x)=2(1-\cos (x)) .
$$

Therefore, the coarse grid correction symbol $C G C$ is of the form

$$
\begin{aligned}
C G C(x) & =I_{2}-\left(\begin{array}{l}
1 \\
1
\end{array}\right)\left(\begin{array}{ll}
1 & 1
\end{array}\right)\left(\begin{array}{cc}
2 & -\alpha \\
-\bar{\alpha} & 2
\end{array}\right) /(2-\exp (i x)-\exp (-i x)) \\
& =\frac{1}{2-\exp (i x)-\exp (-i x)}\left(\begin{array}{c}
1-\exp (i x) \\
-1+\exp (-i x)
\end{array}\right)\left(\begin{array}{ll}
1 & -1
\end{array}\right),
\end{aligned}
$$

again a rank-1 matrix. But in this case the remaining rank-1 $C G C$ symbol still has the singularity induced by $f(x)$. If we are able to find smoothers that give smoothing corrections orthogonal to $C G C(x)$, then also the singularity vanishes at least in the symbol. For presmoothing from the right we have to satisfy

or

$$
\left(\begin{array}{ll}
1 & -1
\end{array}\right) \cdot\left(I_{2}-M_{r}^{-1}\left(\begin{array}{cc}
2 & -\alpha \\
-\bar{\alpha} & 2
\end{array}\right)\right) \equiv 0
$$

$$
\begin{aligned}
& \left(\begin{array}{ll}
1 & -1
\end{array}\right) M_{r}^{-1}=\left(\begin{array}{ll}
1 & -1
\end{array}\right)\left(\begin{array}{cc}
2 & \alpha \\
\bar{\alpha} & 2
\end{array}\right) /(2-\exp (i x)-\exp (-i x))= \\
& =(1-\exp (-i x) \quad-1+\exp (i x)) /(2-\exp (i x)-\exp (-i x)) .
\end{aligned}
$$

Three special solutions for $M_{r}$ can be described by

$$
M_{r}=\left(\begin{array}{cc}
1-e^{i x} & 0 \\
0 & 1-e^{-i x}
\end{array}\right), M_{r}=\left(\begin{array}{cc}
1 & 0 \\
-1 & 1-e^{-i x}
\end{array}\right), \text { or } M_{r}=\left(\begin{array}{cc}
1-e^{i x} & -1 \\
0 & 1
\end{array}\right) .
$$

The solutions for smoother $N_{r}$ we get by inverting $M_{r}$.

For postsmoother $M_{l}$ the condition $\left(I-M_{l}^{-1} F\right) \cdot C G C(x) \equiv 0$ yields

$$
M_{l}^{-1}\left(\begin{array}{cc}
2 & -\alpha \\
-\bar{\alpha} & 2
\end{array}\right)\left(\begin{array}{c}
1-\exp (i x) \\
-1+\exp (-i x)
\end{array}\right)=\left(\begin{array}{c}
1-\exp (i x) \\
-1+\exp (-i x)
\end{array}\right)
$$

with the special solutions

$$
M_{l}=\left(\begin{array}{cc}
1 & -1 \\
0 & 1-e^{i x}
\end{array}\right), M_{l}=\left(\begin{array}{cc}
1-e^{-i x} & 0 \\
-1 & 1
\end{array}\right), \text { or } M_{l}=\left(\begin{array}{cc}
1-e^{-i x} & 0 \\
0 & 1-e^{i x}
\end{array}\right) .
$$

With these pre-and postsmoothers the symbol $T G S(x)$ will be zero. For the full matrices it turns out that the $n \times n$ matrix $T G S_{n}$ is not zero but of rank 1 in view of the boundary conditions. But we can determine the rank 1 representation of $T G S_{n}$ and thereby determine an additional 'smoother' to eliminate this rank 1 perturbation in order to obtain $T G S_{n}=0$ also for the full $n \times n$ matrix. The combination of

$$
M_{r}=\left(\begin{array}{cc}
1-\exp (i x) & -1 \\
0 & 1
\end{array}\right)=\left(\begin{array}{cc}
-1 & 0 \\
0 & 0
\end{array}\right) \exp (i x)+\left(\begin{array}{cc}
1 & -1 \\
0 & 1
\end{array}\right)
$$


together with left 'smoother' $N_{l}=0.5 \cdot \operatorname{diag}(1,0,1,0, \ldots, 1,0)$ yields $T G S_{n}=0$ for $n=2^{k} \pm 1$. Note, that in this case, the rank-1 representation of $C G C \cdot\left(I-M_{r}^{-1} A\right)$ is given by

$$
C G C \cdot S_{r}:=C G C \cdot\left(I-M_{r}^{-1} A\right)=-\left(\begin{array}{lllll}
1 & 0 & 1 & 0 & \cdots
\end{array}\right)^{T} \cdot\left(\begin{array}{llll}
1 & 0 & 0 & \cdots
\end{array}\right) .
$$

Without additional left 'smoother', $T G S_{n}$ is of rank 1 with slowly growing norm (growing like $O(n)$ ).

By applying only $M_{r}$ without additional smoother $N_{l}$ a Krylov-Multigrid method with a preconditioned conjugate gradient cycle ([15]) would give convergence in 2 steps and could therefore also be considered as a direct solver. Hence, we have shown that with the proper choice of smoother at least in the 1D-case the weak linear prolongation can lead to nearly the same efficiency as the standard prolongation with red-black smoother.

A similar analysis shows that the trivial injection $E$ as prolongation leads to a well-conditioned coarse grid system $A_{c}$ with symbol $C G C$ again of rank 1 . But in this case the smoother has also to remove error components relative to the singular eigenvalue. Therefore, the original Multigrid idea of applying two different methods to reduce the error in two subspaces would be lost if we worked with the trivial injection only.

3. Compact Fourier analysis in the $2 \mathrm{D}$ case. Now we turn to the more interesting $2 \mathrm{D}$ case. We will consider the matrix $A_{5}$ given by the 5 -point stencil and $A_{9}$ given by the 9 -point stencil

$$
A_{5}=\left(\begin{array}{ccc} 
& -1 & \\
-1 & 4 & -1 \\
& -1 &
\end{array}\right), A_{9}=\left(\begin{array}{ccc}
-1 & -1 & -1 \\
-1 & 8 & -1 \\
-1 & -1 & -1
\end{array}\right) \text {. }
$$

First we need the scalar symbols in $x$ and $y$ for a given 2-level Toeplitz matrix $A$ (also called Block Toeplitz Toeplitz Block matrix):

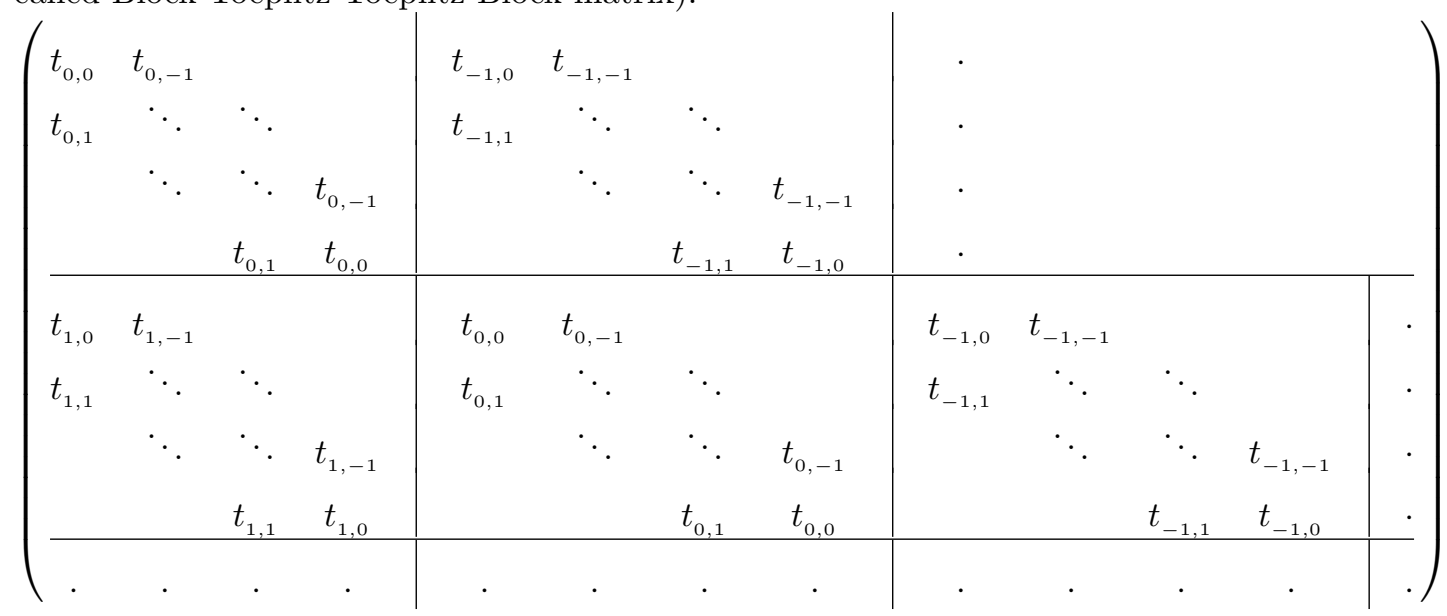

of the form $f(x, y)=\sum_{j, k=-\infty}^{\infty} t_{j, k} e^{i j x+i k y}$. For $A_{5}$ we get

$$
f_{5}(x, y)=4-\exp (i x)-\exp (-i x)-\exp (i y)-\exp (-i y)=2(2-\cos (x)-\cos (y))
$$

and for $A_{9}$

$$
\begin{aligned}
f_{9}(x, y)= & 8-\exp (i x)-\exp (-i x)-\exp (i y)-\exp (-i y)- \\
& -\exp (i(x-y))-\exp (i(y-x))-\exp (i(x+y)-\exp (-i(x+y))= \\
= & 8-2 \cos (x)-2 \cos (y)-2 \cos (x+y)-2 \cos (x-y) \\
= & 8-2 \cos (x)-2 \cos (y)-4 \cos (x) \cos (y) .
\end{aligned}
$$


The bilinear interpolation is related to the Kronecker product of the 1D projection $B=\operatorname{tridiag}(1,2,1) \otimes \operatorname{tridiag}(1,2,1)$ with scalar symbol

$$
b(x, y)=b(x) b(y)=(1+\cos (x))(1+\cos (y)) .
$$

To obtain the block symbols for these functions we have to apply the $1 \mathrm{D}$ technique developed in (2.1) and (2.2) in two steps, first relative to $x$, and then relative to $y$ :

$$
h(x, y) \rightarrow H_{x}(x, y) \rightarrow H_{x y}(x, y)=H(x, y) .
$$

Here $H_{x}(x, y)$ is a $2 \times 2$ matrix function derived by the $1 \mathrm{D}$ step in $x$, and $H(x, y)$ is a $4 \times 4$ matrix function by the 1D step in $y$ applied on $H_{x}$.

For the 5-point stencil with symbol $f_{5}(x, y)=4-2 \cos (x)-2 \cos (y)$ the block symbol relative to $x$ is

$$
F_{x}(x, y)=\left(\begin{array}{cc}
4-2 \cos (y) & -2 e^{i x / 2} \cos (x / 2) \\
-2 e^{-i x / 2} \cos (x / 2) & 4-2 \cos (y)
\end{array}\right),
$$

and the second transformation relative to $y$ gives

$$
\begin{gathered}
F(x, y)=\left(\begin{array}{ccc}
\frac{1}{2}\left(F_{x}\left(x, \frac{y}{2}\right)+F_{x}\left(x, \frac{y}{2}+\pi\right)\right) & \frac{1}{2} e^{i y / 2}\left(F_{x}\left(x, \frac{y}{2}\right)-F_{x}\left(x, \frac{y}{2}+\pi\right)\right) \\
\frac{1}{2} e^{-i y / 2}\left(F_{x}\left(x, \frac{y}{2}\right)-F_{x}\left(x, \frac{y}{2}+\pi\right)\right) & \frac{1}{2}\left(F_{x}\left(x, \frac{y}{2}\right)+F_{x}\left(x, \frac{y}{2}+\pi\right)\right)
\end{array}\right)= \\
=\left(\begin{array}{cccc}
4 & -1-e^{i x} & -1-e^{i y} & 0 \\
-1-e^{-i x} & 4 & 0 & -1-e^{i y} \\
-1-e^{-i y} & 0 & 4 & -1-e^{i x} \\
0 & -1-e^{-i y} & -1-e^{-i x} & 4
\end{array}\right)=\left(\begin{array}{cccc}
4 & -\alpha & -\beta & 0 \\
-\bar{\alpha} & 4 & 0 & -\beta \\
-\bar{\beta} & 0 & 4 & -\alpha \\
0 & -\bar{\beta} & -\bar{\alpha} & 4
\end{array}\right)
\end{gathered}
$$

with $\alpha=1+\exp (i x)$ and $\beta=1+\exp (i y)$. Again the index 1 in the symbol is related to the points that are fine and coarse in both dimensions while the index 4 is related to the points that are only fine and noncoarse in both dimensions.

To derive a $2 \mathrm{D}$ block symbol $H(x, y)$ directly, in a first step we replace the scalar $n \times n$ Toeplitz block matrices in the $n^{2} \times n^{2}$ matrix $A$ by symbols in $x$, resulting in a $2 n \times 2 n$ matrix. In a following step we replace these $n \times n$ Toeplitz blocks by symbols in $y$. For the 5-point stencil with matrix $A_{5}=$

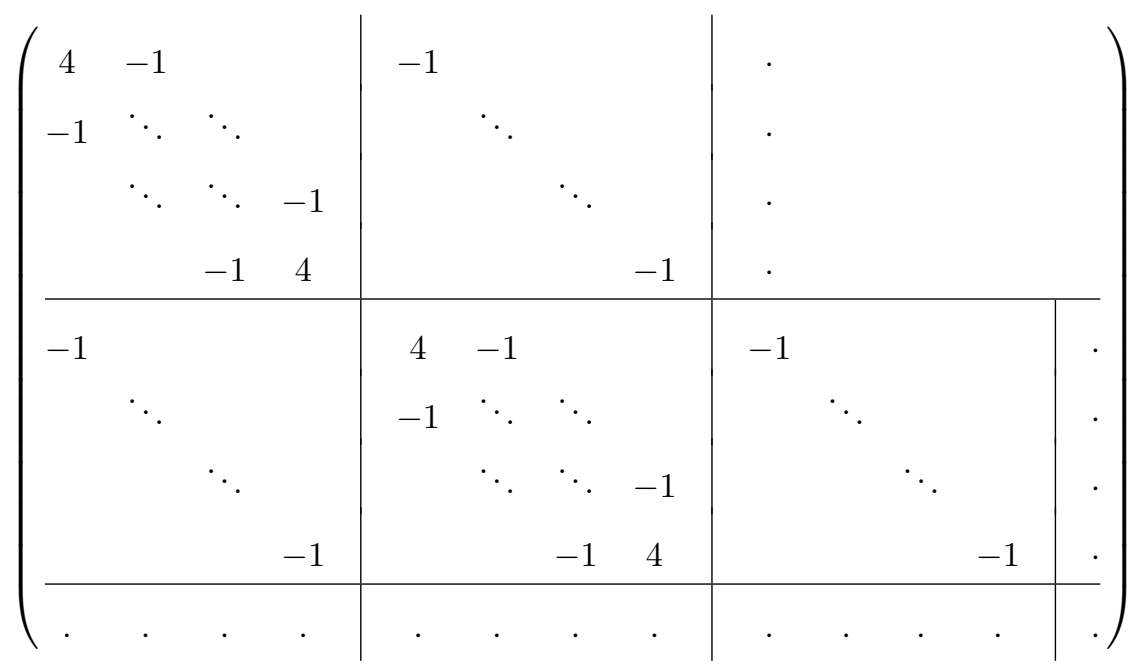


the first step replacing the blocks by their symbols in $x$ leads to

$=\left(\begin{array}{cc|cc|cc|c}4 & -1-e^{i x} & -1 & & & \\ -1-e^{-i x} & 4 & & -1 & . & \\ \hline-1 & & 4 & -1-e^{i x} & -1 & \\ & -1 & -1-e^{-i x} & 4 & & -1 & \cdot \\ \hline \cdot & \cdot & . & \cdot & . & \cdot\end{array}\right)$,

and the second step with respect to $y$ gives

$$
F_{5}(x, y)=\left(\begin{array}{cc|cc}
4 & -1-e^{i x} & -1-e^{i y} & 0 \\
-1-e^{-i x} & 4 & 0 & -1-e^{i y} \\
\hline-1-e^{-i y} & 0 & 4 & -1-e^{i x} \\
0 & -1-e^{-i y} & -1-e^{-i x} & 4
\end{array}\right) .
$$

Note, that we can recover the original matrix $A$ from the block symbol by going backwards from (3.4) via (3.3) to (3.2). For $A_{5}$ the two-level odd-even permutation in the $2 \mathrm{D}$ case can be derived directly by the block symbol and is given by the block matrix

$$
\left(\begin{array}{cccc}
4 \cdot I \otimes I & -T(\alpha) \otimes I & -I \otimes T(\beta) & 0 \\
-T(\bar{\alpha}) \otimes I & 4 \cdot I \otimes I & 0 & -I \otimes T(\beta) \\
-I \otimes T(\bar{\beta}) & 0 & 4 \cdot I \otimes I & -T(\alpha) \otimes I \\
0 & -I \otimes T(\bar{\beta}) & -T(\bar{\alpha}) \otimes I & 4 \cdot I \otimes I
\end{array}\right)
$$

In the same way the block symbol for the 9-point stencil results as

$$
F_{9}(x, y)=\left(\begin{array}{cccc}
8 & -\alpha & -\beta & -\alpha \beta \\
-\bar{\alpha} & 8 & -\bar{\alpha} \beta & -\beta \\
-\bar{\beta} & -\alpha \bar{\beta} & 8 & -\alpha \\
-\bar{\alpha} \bar{\beta} & -\bar{\beta} & -\bar{\alpha} & 8
\end{array}\right)
$$

and the standard prolongation (3.1) as

$$
B(x, y)=\left(\begin{array}{cccc}
4 & 2 \alpha & 2 \beta & \alpha \beta \\
2 \bar{\alpha} & 4 & \bar{\alpha} \beta & 2 \beta \\
2 \bar{\beta} & \alpha \bar{\beta} & 4 & 2 \alpha \\
\bar{\alpha} \bar{\beta} & 2 \bar{\beta} & 2 \bar{\alpha} & 4
\end{array}\right) .
$$

The description of the Twogrid step is similar to the 1D case, only the projection is given by picking out every second entry in $x$ and $y$, resp. every second column/row and block column/block row in $A$. As in the $1 \mathrm{D}$ case, the error reduction $T G S$ is obtained by combining the pre- and postsmoothing and the coarse grid correction (1.3) and (2.5) in the form

$$
T G S=\left(I-M_{l}^{-1} A\right)\left(I-\left(B E^{T} A_{c}^{-1} E B\right) A\right)\left(I-M_{r}^{-1} A\right)=S_{l} \cdot C G C \cdot S_{r} .
$$

Remark 2: If we are interested in analyzing the smoother $S=I-M^{-1} A$ alone, we can again use the symbols for $M$ and $A$ in the form $I-M^{-1}(x, y) F(x, y)$. In 
order to get information about the smoothing behavior relative to the high frequency subspace we can analyze the symbol restricted on the eigenspaces relative to the three eigenvalues that are nonsingular. We can transform $F$ by the diagonal matrix $D=\operatorname{diag}(1, \alpha /|\alpha|, \beta /|\beta|, \alpha \beta /|\alpha \beta|)$ to a real symmetric block circulant $4 \times 4$ matrix

$$
\left(\begin{array}{cccc}
4 & -|\alpha| & -|\beta| & 0 \\
-|\alpha| & 4 & 0 & -|\beta| \\
-|\beta| & 0 & 4 & -|\alpha| \\
0 & -|\beta| & -|\alpha| & 4
\end{array}\right)
$$

with eigenvectors given by $F_{2} \otimes F_{2}, F_{2}$ the Fourier matrix. Therefore, we have the eigenvector $(1,1,1,1)^{T}$ corresponding to the eigenvalue $4-|\alpha|-|\beta|=f(x / 2, y / 2)$ which has a singularity at $x=0$. Hence, the high frequency subspace is spanned by the remaining three eigenvectors to eigenvalues $f(x / 2+\pi, y / 2), f(x / 2, y / 2+\pi)$ and $f(x / 2+\pi, y / 2+\pi)$. The smoothing behavior of $S$ can be determined by the subspace relative to these 3 eigenvalues. The minimum eigenvalue has values in $[0,4]$, the remaining three nonzero eigenvalues in $[2,8]$. Therefore, the reduction to the subspace of the three nonzero eigenvalues is related to the subspace to all eigenvalues in the range $[4,8]$.

For the 9-point stencil the same transformation with $D$ gives again a block circulant matrix with first row

$$
\left(\begin{array}{llll}
8 & -|\alpha| & -|\beta| & -|\alpha \beta|
\end{array}\right) .
$$

The minimum eigenvalue has values in $[0,8]$, while the three nonzero eigenvalues range in $[6,12]$. The eigenspace relative to the three eigenvectors is the same as in the 5 -point stencil.

Again let us display the symbols for the standard smoothers. The lower triangular Gauss-Seidel smoother is related to the symbols

$$
L_{G S_{5}}=\left(\begin{array}{cccc}
4 & -e^{i x} & -e^{i y} & 0 \\
-1 & 4 & 0 & -e^{i y} \\
-1 & 0 & 4 & -e^{i x} \\
0 & -1 & -1 & 4
\end{array}\right), L_{G S_{9}}=\left(\begin{array}{cccc}
8 & -e^{i x} & -e^{i y} & -e^{i y} \alpha \\
-1 & 8 & -\bar{\alpha} e^{i y} & -e^{i y} \\
-1 & -\alpha & 8 & -e^{i x} \\
-\bar{\alpha} & -1 & -1 & 8
\end{array}\right) .
$$

Standard block Gauss-Seidel for $A_{5}$ and $A_{9}$ are related to the symbols

$$
L_{B G S_{5}}=\left(\begin{array}{cccc}
4 & -\alpha & -e^{i y} & 0 \\
-\bar{\alpha} & 4 & 0 & -e^{i y} \\
-1 & 0 & 4 & -\alpha \\
0 & -1 & -\bar{\alpha} & 4
\end{array}\right), L_{B G S_{9}}=\left(\begin{array}{cccc}
8 & -\alpha & -e^{i y} & -e^{i y} \alpha \\
-\bar{\alpha} & 8 & -\bar{\alpha} e^{i y} & -e^{i y} \\
-1 & -\alpha & 8 & -\alpha \\
-\bar{\alpha} & -1 & -\bar{\alpha} & 8
\end{array}\right)
$$

Furthermore, we can represent smoothers that order the grid points after their character in coarse in both dimension, mixed or non-coarse in both dimensions. Based on the symbol $F$ we derive the four-color Gauss-Seidel smoother related to $\operatorname{tril}(F(x, y)):=$ $L_{\tilde{G S}}$. In $L_{\tilde{G S}}$ we use the original ordering of the four colors in coarse-coarse, coarsenoncoarse, noncoarse-coarse, noncoarse-noncoarse. Changing the ordering in $F(x, y)$ such that indices coarse and non-coarse in both dimensions come first (by permuting index 2 with 4 ) leads for $A_{5}$ to the red-black Gauss-Seidel smoother:

$$
L_{R B}=\left(\begin{array}{cccc}
4 & 0 & -\beta & -\alpha \\
0 & 4 & -\bar{\alpha} & -\bar{\beta} \\
0 & 0 & 4 & 0 \\
0 & 0 & 0 & 4
\end{array}\right)
$$


On the other hand, the block triangular part of the symbol gives a four-color block Gauss-Seidel smoother

$$
L_{B \tilde{G} S_{5}}:=\left(\begin{array}{cccc}
4 & -\alpha & 0 & 0 \\
-\bar{\alpha} & 4 & 0 & 0 \\
-\bar{\beta} & 0 & 4 & -\alpha \\
0 & -\bar{\beta} & -\bar{\alpha} & 4
\end{array}\right) \quad, \quad L_{B \tilde{G} S_{9}}:=\left(\begin{array}{cccc}
8 & -\alpha & 0 & 0 \\
-\bar{\alpha} & 8 & 0 & 0 \\
\bar{\beta} & -\alpha \bar{\beta} & 8 & -\alpha \\
-\bar{\alpha} \bar{\beta} & -\bar{\beta} & -\bar{\alpha} & 8
\end{array}\right)
$$

3.1. Error reduction for the bilinear interpolation. First we have to determine the symbol of the coarse problem derived by the Galerkin projection

$$
\begin{aligned}
\left(f_{5}\right)_{c}(x, y) & =B_{1} F_{5} B_{1}^{H}=\left(\begin{array}{llll}
4 & 2 \alpha & 2 \beta & \alpha \beta
\end{array}\right)\left(\begin{array}{cccc}
4 & -\alpha & -\beta & 0 \\
-\bar{\alpha} & 4 & 0 & -\beta \\
-\bar{\beta} & 0 & 4 & -\alpha \\
0 & -\bar{\beta} & -\bar{\alpha} & 4
\end{array}\right)\left(\begin{array}{c}
4 \\
2 \bar{\alpha} \\
2 \bar{\beta} \\
\bar{\alpha} \bar{\beta}
\end{array}\right)= \\
& =64-4|\alpha|^{2}|\beta|^{2}=16(3-\cos (x) \cos (y)-\cos (x) \cos (y))= \\
& =8(6-2 \cos (x)-2 \cos (y)-\cos (x-y)-\cos (x+y))
\end{aligned}
$$

and

$$
\begin{aligned}
& \text { and } \\
& \qquad \begin{array}{l}
\left(f_{9}\right)_{c}(x, y)=B_{1} F_{9} B_{1}^{H}=\left(\begin{array}{llll}
4 & 2 \alpha & 2 \beta & \alpha \beta
\end{array}\right)\left(\begin{array}{cccc}
8 & -\alpha & -\beta & -\alpha \beta \\
-\bar{\alpha} & 8 & -\bar{\alpha} \beta & -\beta \\
-\bar{\beta} & -\alpha \bar{\beta} & 8 & -\alpha \\
-\bar{\alpha} \bar{\beta} & -\bar{\beta} & -\bar{\alpha} & 8
\end{array}\right)\left(\begin{array}{c}
4 \\
2 \bar{\alpha} \\
2 \bar{\beta} \\
\bar{\alpha} \bar{\beta}
\end{array}\right)= \\
=16(8-2 \cos (x)-2 \cos (y)-4 \cos (x) \cos (y))=16 f_{9}(x, y)=16\left(8+|\alpha|^{2}+|\beta|^{2}-|\alpha \beta|^{2}\right) .
\end{array}
\end{aligned}
$$

Note, that the coarse Galerkin projection for $f_{5}$ has the same zero as $f_{5}$ but is essentially different in the sense that the quotient is not a constant factor. Therefore, with this combination of discretization and prolongation, a direct solver could be derived only for a Twogrid method but not for a recursively defined Multigrid algorithm.

Next, we can determine the symbols for $C G C_{5}(x, y)=I_{4}-B_{1}^{H}\left(f_{5}\right)_{c}^{-1} B_{1} F_{5}=$

$$
\begin{aligned}
& =I_{4}-\left(\begin{array}{c}
4 \\
2 \bar{\alpha} \\
2 \bar{\beta} \\
\bar{\alpha} \bar{\beta}
\end{array}\right)\left(\begin{array}{llll}
4 & 2 \alpha & 2 \beta & \alpha \beta
\end{array}\right)\left(\begin{array}{cccc}
4 & -\alpha & -\beta & 0 \\
-\bar{\alpha} & 4 & 0 & -\beta \\
-\bar{\beta} & 0 & 4 & -\alpha \\
0 & -\bar{\beta} & -\bar{\alpha} & 4
\end{array}\right) /\left(f_{5}\right)_{c}= \\
& =\frac{1}{\left(f_{5}\right)_{c}}\left(\begin{array}{cccc}
8|\alpha|^{2}+8|\beta|^{2}-4|\alpha \beta|^{2} & 4 \alpha\left(-4+|\beta|^{2}\right) & 4 \beta\left(-4+|\alpha|^{2}\right) & 0 \\
4 \bar{\alpha}\left(-8+|\alpha|^{2}+|\beta|^{2}\right) & 64-8|\alpha|^{2}-2|\alpha \beta|^{2} & 2 \bar{\alpha} \beta\left(-4+|\alpha|^{2}\right) & 0 \\
4 \bar{\beta}\left(-8+|\alpha|^{2}+|\beta|^{2}\right) & 2 \alpha \bar{\beta}\left(-4+|\beta|^{2}\right) & 64-8|\beta|^{2}-2|\alpha \beta|^{2} & 0 \\
2 \bar{\alpha} \bar{\beta}\left(-8+|\alpha|^{2}+|\beta|^{2}\right) & \bar{\beta}|\alpha|^{2}\left(-4+|\beta|^{2}\right) & \bar{\alpha}|\beta|^{2}\left(-4+|\alpha|^{2}\right) & 64-4|\alpha \beta|^{2}
\end{array}\right)
\end{aligned}
$$

and $C G C_{9}(x, y)=I_{4}-B_{1}^{H}\left(f_{9}\right)_{c}^{-1} B_{1} F_{9}=$

$$
\begin{gathered}
=\frac{1}{\left(f_{9}\right)_{c}}\left(\left(\begin{array}{cccc}
-12|\alpha \beta|^{2} & 0 & 0 & 0 \\
2 \bar{\alpha}|\alpha \beta|^{2} & 128-10|\alpha \beta|^{2} & 0 & 0 \\
2 \bar{\beta}|\alpha \beta|^{2} & 0 & 128-10|\alpha \beta|^{2} & 0 \\
\bar{\alpha} \bar{\beta}|\alpha \beta|^{2} & 0 & 0 & 128-16|\alpha \beta|^{2}
\end{array}\right)+\right. \\
\left.\left(\begin{array}{cccc}
24\left(|\alpha|^{2}+|\beta|^{2}\right) & 12 \alpha\left(-4+|\beta|^{2}\right) & 12 \beta\left(-4+|\alpha|^{2}\right) & 0 \\
4 \bar{\alpha}\left(-16+|\alpha|^{2}+|\beta|^{2}\right) & -8|\alpha|^{2}+16|\beta|^{2} & 6 \bar{\alpha} \beta\left(-4+|\alpha|^{2}\right) & 0 \\
4 \bar{\beta}\left(-16+|\alpha|^{2}+|\beta|^{2}\right) & 6 \alpha \bar{\beta}\left(-4+|\beta|^{2}\right) & 16|\alpha|^{2}-8|\beta|^{2} & 0 \\
2 \bar{\alpha} \bar{\beta}\left(-16+|\alpha|^{2}+|\beta|^{2}\right) & 3 \bar{\beta}|\alpha|^{2}\left(-4+|\beta|^{2}\right) & 3 \bar{\alpha}|\beta|^{2}\left(-4+|\alpha|^{2}\right) & 16\left(|\alpha|^{2}+|\beta|^{2}\right)
\end{array}\right)\right) .
\end{gathered}
$$

For these $C G C$ symbols we want to analyze the smoothing behavior and the total Twogrid error reduction factor. For the total error reduction factor we consider for 
$T G S(x, y)=S_{l} \cdot C G C \cdot S_{r}$ with different pre- and postsmoother the 2-norm and the spectral radius. For a smoothing analysis we reduce $S=I-M^{-1} A$ to the subspace relative to the three nonzero eigenvalues of $F$ and compute again the norm and the spectral radius.

For deriving an efficient smoother, we first note that with presmmoother only the Twogrid error can be written as

$$
S_{l} \cdot C G C=\left(I-M^{-1} F\right) \cdot C G C=M^{-1}(M-F) \cdot C G C .
$$

Therefore, a rank reducing smoother can be defined by choosing for $M$ a regular submatrix of $F$, e.g. $M$ equals $F$ except for the first (or last) row (or column). In the ideal case the one-dimensional left sided kernel of $C G C$ would coincide with the one-dimensional range of $M-F$ giving $T G S(x, y)=0$. Unfortunately, this can be derived only in very special cases. Nevertheless, the rank reducing smoother leads to a rank-1 matrix $S_{l} \cdot C G C$ of small norm. If the $C G C$-symbol is of special form, e.g. with first row zero, then the rank reducing smoother changing only the first column of $M$ would give $S_{l} \cdot C G C=0$. For the presmoother applied on the right hand side the same idea leads to the condition

$$
C G C \cdot(I-N F)=C G C \cdot\left(F^{-1}-N\right) F \text { or } C G C \cdot\left(F^{-1}-M^{-1}\right) F,
$$

which is not so easy to satisfy. The advantage of rank reducing smoothers that coincide with large subparts of $F$ is that the total residual is reduced to a subspace of a quarter of the full space. The resulting smoother is well-conditioned but cannot be solved directly because it preserves mainly the sparsity structure of $A$. So an additional iterative method like preconditioned conjugate gradient method (pcg) is necessary in order to solve the linear system related to the smoothing iteration.

Next we consider the Jacobi smoother $J(\omega)=I-\omega F / 4$ or two combined steps of the form $J\left(\omega_{1}, \omega_{2}\right)=J\left(\omega_{1}\right) J\left(\omega_{2}\right)$. Furthermore, we analyze standard Gauss-Seidel and Block Gauss-Seidel, the four-color Gauss Seidel $L_{\tilde{G S}}=\operatorname{tril}(F)$ and Block GaussSeidel $L_{B \tilde{G} S}$ related to the symbol, and the red-black smoother $L_{R B}$ related to the permutation $j=\left[\begin{array}{llll}1 & 4 & 3 & 2\end{array}\right]$ and the blocking of $F(j, j)$ in two-by-two blocks. Often Gauss-Seidel smoothers are applied symmetrically in the form $\left(I-L^{-1} F\right)\left(I-L^{-T} F\right)$. In the sequel, we will also consider modifications where we allow more general combinations of different smoothers. As new rank reducing smoother we introduce

$$
F_{c 1}:=\left(\begin{array}{cccc}
4 & -\alpha & -\beta & 0 \\
0 & 4 & 0 & -\beta \\
0 & 0 & 4 & -\alpha \\
0 & -\bar{\beta} & -\bar{\alpha} & 4
\end{array}\right) \quad, \quad F_{c 12}:=\left(\begin{array}{cccc}
4 & -\alpha & -\beta & 0 \\
0 & 4 & 0 & -\beta \\
0 & 0 & 4 & -\alpha \\
0 & 0 & -\bar{\alpha} & 4
\end{array}\right) .
$$

Deleting in the symbol all entries in the first column except for the diagonal entry is related to deleting in the full matrix in every second column of every second column block all entries except for the diagonal entry, starting with the first column in the first column block. Note that the first column in the symbol is related to grid points that belong to the fine and coarse grid in both dimensions, while the fourth column is related to grid points only fine in both dimensions. Similarly, we can define e.g. $F_{c 4}$, $F_{r 1}, F_{r 4}$, related to the last column, first and last row of $F$ respectively, and $F_{c 43}$, $F_{r 12}$, and $F_{r 43}$ with an additional zero in the nondiagonal neighboring block.

$F_{c 12}$ leads only to a rank-2 matrix $S_{l} \cdot C G C$, but $F_{c 12}$ is a block triangular submatrix of (3.5), so linear systems with $F_{c 12}$ can be solved much easier than with $F_{c 1}$. The same is true for all other modifications $F_{c i j}$ and $F_{r i j}$. The costs for computing one smoothing step with e.g. $F_{c 12}$ are nearly the same as the costs for one step with standard Gauss-Seidel or colored Gauss-Seidel $L_{\tilde{G S}}$; we can use the splitting $F=F_{c 12}+U$ 
FIG. 3.1. Sparsity pattern of $F_{c 12}$ as submatrix of $A$ of size $6^{2} \times 6^{2}$ in original ordering and in four color ordering. Obviously, for solving a matrix with such a pattern the only involved part is solving tridiagonal submatrices.

TABLE 3.1

Smoothing factor computed based on the block symbol for different smoothers (first row: norm, second row: spectral radius) for 5-point stencil. The Gauss-Seidel smoothers are applied symmetrically $\left(I-L^{-1} F\right)\left(I-L^{-T} F\right)$ and the last column is related to $\left(I-F_{c 43}^{-1} F\right)\left(I-F_{r 12}^{-1} F\right)$.

\begin{tabular}{c|c|c|c|c|c|c|c|c|c|c}
$J(.8)$ & $J(.8, .8)$ & $J\left(\omega_{1}, \omega_{2}\right)$ & $G S$ & $B G S$ & $\tilde{G S}$ & $B \tilde{G} S$ & $R B$ & $F_{c 1}$ & $F_{c 12}$ & $F_{c 43}, F_{r 12}$ \\
\hline 0.60 & 0.36 & 0.2195 & 0.17 & 0.17 & 0.20 & 0.27 & 0.27 & 0.35 & 0.55 & 0.14 \\
\hline 0.60 & 0.36 & 0.2195 & 0.13 & 0.12 & 0.13 & 0.12 & 0.24 & 0.07 & 0.24 & 0.058
\end{tabular}

resulting in the smoothing step $F_{c 12} x^{k+1}=b-U x^{k}$ where the upper triangular part $U$ contains less entries than in the Gauss-Seidel splitting but on the other side the solution of the linear system in $F_{c 12}$ is slightly more expensive than the solution of the triangular Gauss-Seidel matrix. So in total the costs are nearly the same (upto $10 \%$ more). On the other side the smoothing factor and the Twogrid error reduction are more than a factor 2 better compared to e.g. Gauss-Seidel type smoothers. For a description of the pattern of the full matrix related to $F_{c 12}$ see Fig. 3.1. Like the red-black smoother, linear systems in $F_{c 12}$ can be implemented efficiently in parallel.

Note, that the matrix $F_{c 1}$ is well-conditioned, but solving linear equations with $F_{c 1}$ cannot be done directly, but only iteratively. For this aim, we have to consider the subblock $A_{24}:=F(2: 4,2: 4)$ and solve $A_{24}^{-1} x$ e.g. by pcg with preconditioner $L_{A} L_{A}^{T}$ and $L_{A}:=\operatorname{tril}\left(A_{24}\right)$. Then the condition number of the preconditioned $A_{24}$ is less than 2 , so we can expect very fast convergence.

For the rank reducing smoother derived from the symbol we have some nice properties. The inverse can be often derived explicitly, so we can also use the inverse as approximate inverse smoother, that is much more efficient in parallel. For example, for the 5-point stencil it holds

$$
L_{\tilde{G S}}^{-1}=\frac{1}{16}\left(\begin{array}{cccc}
4 & & \\
\bar{\alpha} & 4 & & \\
\bar{\beta} & 0 & 4 & \\
\bar{\alpha} \bar{\beta} / 8 & \bar{\beta} & \bar{\alpha} & 4
\end{array}\right) \text {, and } L_{R B}^{-1}=\frac{1}{16}\left(\begin{array}{cccc}
4 & 0 & & \\
0 & 4 & & \\
\bar{\beta} & \alpha & 4 & 0 \\
\bar{\alpha} & \beta & 0 & 4
\end{array}\right) \text {. }
$$

Applying a rank reducing smoother $F_{c 1} \ldots, F_{r 4}, F_{c 12}, \ldots, F_{r 43}, \tilde{G S}$ or $B \tilde{G} S$ from the left always leads to a sparsity pattern in $M_{l}-F$ that can be adjusted to the sparsity pattern of $C G C(x, y)$ resulting in a small norm of $T G S(x, y)$. For presmoother in view of $C G C \cdot\left(I-M_{r}^{-1} F\right)=C G C \cdot M_{r}^{-1}\left(M_{r}-F\right)$ this is only true for the column oriented rank reducing smoothers $F_{c 1}, F_{c 4}, F_{c 12}, F_{c 43}, \tilde{G S}$, and $B \tilde{G} S$.

In the tables 3.1 and 3.2 we display the smoothing factor and the error reduction in a Twogrid step for various smoothers. Note, that - if not stated otherwise - all the numerical values are related to block symbols, e.g. $T G S(x, y)$, maximizing over all $x$. For two-step Jacobi smoothing the optimal choice for $\left(\omega_{1}, \omega_{2}\right)$ is $8 /(10 \pm 3 \sqrt{2})$ with smoothing factor $9 / 41 \approx 0.21951$. The four-color Gauss-Seidel $L_{\tilde{G S}}$ with the ordering coarse-coarse first and noncoarse-noncoarse last is slightly better than the red-black Gauss-Seidel. In tables $3.3-3.4$ we will compare the norm of the symbol $T G S(x, y)$ and of the full matrix $T G S_{n^{2}}$ for different smoothers. Furthermore, for chosen $L$ we consider different orderings of $L$ and $L^{T}$ in the post- and presmoother. The results show that the symbol reflects the behavior of the full matrix, and that the ordering may change the behavior dramatically for colored Gauss-Seidel smoother. 
TABLE 3.2

Maximum norm and spectral radius of $T G S(x, y)=S_{l} \cdot C G C \cdot S_{r}$ with different smoothers (first row: norm, second row: spectral radius) for 5-point stencil. Here, $*$ in $S_{l}, S_{r}$ denotes that the same smoother is applied as pre- and postsmoother.

\begin{tabular}{c|c|c|c|c|c|c|c|c}
$S_{J(.8), *}$ & $S_{J(.8,8), *}$ & $S_{J\left(\omega_{1}, \omega_{2}\right), *}$ & $S_{G S, *}$ & $S_{B G S, *}$ & $S_{R B, *}$ & $S_{F_{c 4}}, S_{F_{r 1}}$ & $S_{F_{c 43}}^{2}, S_{F_{r 12}}^{2}$ & $S_{F_{c 12}, *}^{2}$ \\
\hline 0.36 & 0.13 & 0.13 & 0.056 & 0.10 & 0.15 & 0.068 & 0.048 & 0.025 \\
\hline 0.36 & 0.13 & 0.083 & 0.054 & 0.045 & 0.12 & 0.067 & 0.027 & 0.016
\end{tabular}

TABLE 3.3

Norm of TGS $(x, y)$ and of the $n^{2} \times n^{2}$ matrix $T G S_{n^{2}}=S_{l} \cdot C G C \cdot S_{r}, n=40$, for smoother $\tilde{G S}$ (first row: symbol, second row: full matrix) for 5-point stencil applying the smoothers in different ordering in $S_{l}$ and $S_{r}$.

\begin{tabular}{c||c|c|c|c|c|c} 
& $L^{\prime} L^{\prime}, L^{\prime} L^{\prime}$ & $L L, L L$ & $L^{\prime} L, L^{\prime} L$ & $L L^{\prime}, L L^{\prime}$ & $L L^{\prime}, L^{\prime} L$ & $L^{\prime} L, L L^{\prime}$ \\
\hline $\max _{x, y}\|T G S(x, y)\|_{2}$ & 0.029 & 0.032 & 0.20 & 0.13 & 0.064 & 0.31 \\
\hline$\left\|T G S_{n^{2}}\right\|_{2}$ & 0.027 & 0.030 & 0.20 & 0.13 & 0.062 & 0.30
\end{tabular}

For the standard Gauss-Seidel smoother the ordering of $L$ and $L^{T}$ does not affect the norm of the Twogrid step. In this case the error reduction is given by $\left\|T G S_{n^{2}}\right\|_{2}=$ 0.055 for the full matrix and $\max _{x, y}\|T G S(x, y)\|_{2}=0.056$ for the symbol. The rank reducing smoother $F_{c 12}$, applied twice as post- and presmoother, gives the best result $\max _{x, y}\|T G S(x, y)\|_{2}=0.025$.

For the 9-point stencil we get tables 3.5 and 3.6. Note, that for Jacobi smoother the optimal parameter for $J(\omega)$ is $8 / 9$ with smoothing factor $1 / 3$ and for $J\left(\omega_{1}, \omega_{2}\right)$ the optimal pair is $16 /(18 \pm 3 \sqrt{2})$ with smoothing factor $1 / 17 \approx 0.05882$. Optimal results are derived by using the same smoother twice and especially the rank reducing smoother with a rank-2 reduction, e.g. $F_{r 12}$, are optimal with respect to the smoothing factor and the overall error reduction. For the 5-point stencil the optimal overall error reduction $T G S$ for the rank reducing Gauss-Seidel smoother $L_{\tilde{G} S}, L_{B \tilde{G} S}$, resp. $L_{R B}$, takes the values $0.029,0.032$, resp. 0.032 , obtained for the ordering $L^{\prime} L^{\prime}, L^{\prime} L^{\prime}$.

3.2. Twogrid as direct solver for a modified Projection. Here, we consider the standard 5-point stencil, but combined with a nonstandard interpolation. Like the standard projection $b(x, y)=(1+\cos (x))(1+\cos (y))$ an interpolation has to have the zeros $(0, \pi),(\pi, 0)$ and $(\pi, \pi)([7,8,1])$. A simple function with these properties can be derived by considering $\tilde{b}(x, y):=f(x, y+\pi) f(x+\pi, y) f(x+\pi, y+\pi)([13,9,10])$. To obtain the block symbol for $\tilde{b}$ we use the block symbol for the three factors that are only slight modifications of the given function $f_{5}$ :

$$
\begin{aligned}
& \text { (3.6) }\left(\begin{array}{cccc}
4 & \alpha & -\beta & 0 \\
\bar{\alpha} & 4 & 0 & -\beta \\
-\bar{\beta} & 0 & 4 & \alpha \\
0 & -\bar{\beta} & \bar{\alpha} & 4
\end{array}\right)\left(\begin{array}{cccc}
4 & -\alpha & \beta & 0 \\
-\bar{\alpha} & 4 & 0 & \beta \\
\bar{\beta} & 0 & 4 & -\alpha \\
0 & \bar{\beta} & -\bar{\alpha} & 4
\end{array}\right)\left(\begin{array}{cccc}
4 & \alpha & \beta & 0 \\
\bar{\alpha} & 4 & 0 & \beta \\
\bar{\beta} & 0 & 4 & \alpha \\
0 & \bar{\beta} & \bar{\alpha} & 4
\end{array}\right)=\tilde{B}(x, y)= \\
& \left(\begin{array}{cccc}
64-4|\alpha|^{2}-4|\beta|^{2} & \alpha\left(16-|\alpha|^{2}+|\beta|^{2}\right) & \beta\left(16+|\alpha|^{2}-|\beta|^{2}\right) & 8 \alpha \beta \\
\bar{\alpha}\left(16-|\alpha|^{2}+|\beta|^{2}\right) & 64-4|\alpha|^{2}-4|\beta|^{2} & 8 \bar{\alpha} \beta & \beta\left(16+|\alpha|^{2}-|\beta|^{2}\right) \\
\bar{\beta}\left(16+|\alpha|^{2}-|\beta|^{2}\right) & 8 \alpha \bar{\beta} & 64-4|\alpha|^{2}-4|\beta|^{2} & \alpha\left(16-|\alpha|^{2}+|\beta|^{2}\right) \\
8 \bar{\alpha} \bar{\beta} & \bar{\beta}\left(16+|\alpha|^{2}-|\beta|^{2}\right) & \bar{\alpha}\left(16-|\alpha|^{2}+|\beta|^{2}\right) & 64-4|\alpha|^{2}-4|\beta|^{2}
\end{array}\right) .
\end{aligned}
$$

With this prolongation we get the Galerkin coarse grid system 
TABLE 3.4

Norm of $T G S=S_{l} \cdot C G C \cdot S_{r}$ for the symbol and the full $n^{2} \times n^{2}$ matrix, $n=40$, for red-black smoother and the 5-point stencil applying the red-black smoother in different ordering in $S_{l}, S_{r}$.

\begin{tabular}{c||c|c|c|c|c|c} 
& $L^{\prime} L^{\prime}, L^{\prime} L^{\prime}$ & $L L, L L$ & $L^{\prime} L, L^{\prime} L$ & $L L^{\prime}, L L^{\prime}$ & $L L^{\prime}, L^{\prime} L$ & $L^{\prime} L, L L^{\prime}$ \\
\hline $\max _{x, y}\|T G S(x, y)\|_{2}$ & 0.032 & 0.032 & 0.15 & 0.15 & 0.045 & 0.045 \\
\hline$\left\|T G S_{n^{2}}\right\|_{2}$ & 0.030 & 0.030 & 0.15 & 0.15 & 0.043 & 0.043
\end{tabular}

TABLE 3.5

Smoothing factor for different smoothers (first row: norm, second row: spectral radius, computed for $\tilde{S}(x, y)$, the projection of $S(x, y)$ on the subspace corresponding to the larger eigenvalues) for 9-point stencil (all Gauss-Seidel smoother applied symmetrically).

\begin{tabular}{c||c|c|c|c|c|c|c|c|c} 
& $J(8 / 9)$ & $J(8 / 9,8 / 9)$ & $J\left(\omega_{1}, \omega_{2}\right)$ & $G S$ & $B G S$ & $G S$ & $B \tilde{G} S$ & $F_{c 4}$ & $F_{c 43}, F_{r 12}$ \\
\hline$\|\tilde{S}(x, y)\|_{2}$ & 0.33 & 0.11 & 0.0588 & 0.14 & 0.17 & 0.08 & 0.075 & 0.17 & 0.033 \\
\hline$\rho(\tilde{S}(x, y))$ & 0.33 & 0.11 & 0.0588 & 0.10 & 0.11 & 0.07 & 0.045 & 0.10 & 0.031
\end{tabular}

$$
\begin{gathered}
\tilde{f}_{c}=\left(\begin{array}{c}
64-4|\alpha|^{2}-4|\beta|^{2} \\
\alpha\left(16-|\alpha|^{2}+|\beta|^{2}\right) \\
\beta\left(16+|\alpha|^{2}-|\beta|^{2}\right) \\
8 \alpha \beta
\end{array}\right)\left(\begin{array}{cccc}
4 & -\alpha & -\beta & 0 \\
-\bar{\alpha} & 4 & 0 & -\beta \\
-\bar{\beta} & 0 & 4 & -\alpha \\
0 & -\bar{\beta} & -\bar{\alpha} & 4
\end{array}\right)\left(\begin{array}{c}
64-4|\alpha|^{2}-4|\beta|^{2} \\
\bar{\alpha}\left(16-|\alpha|^{2}+|\beta|^{2}\right) \\
\bar{\beta}\left(16+|\alpha|^{2}-|\beta|^{2}\right) \\
8 \bar{\alpha} \bar{\beta}
\end{array}\right)= \\
=16384-3072|\alpha|^{2}-3072|\beta|^{2}+192|\alpha|^{4}+192|\beta|^{4}+128|\alpha \beta|^{2}-4|\alpha|^{6}-4|\beta|^{6}+4|\alpha|^{4}|\beta|^{2}+4|\alpha|^{2}|\beta|^{4}
\end{gathered}
$$

and the symbol for the coarse grid correction

$$
C G C(x, y)=I-B_{1}^{H} B_{1} F / \tilde{f}_{c}=\left(\begin{array}{cccc}
0 & 0 & 0 & 0 \\
g_{\alpha} & \tilde{f}_{c} & 0 & 0 \\
g_{\beta} & 0 & \tilde{f}_{c} & 0 \\
g_{\alpha \beta} & 0 & 0 & \tilde{f}_{c}
\end{array}\right) / \tilde{f}_{c}
$$

with

$$
\begin{aligned}
g_{\alpha} & =\bar{\alpha}\left(-4096+768|\alpha|^{2}+256|\beta|^{2}-48|\alpha|^{4}+32|\alpha \beta|^{2}+16|\beta|^{4}+|\alpha|^{6}-|\beta|^{6}-3|\alpha|^{4}|\beta|^{2}+3|\alpha|^{2}|\beta|^{4}\right), \\
g_{\beta} & =\bar{\beta}\left(-4096+768|\beta|^{2}+256|\alpha|^{2}-48|\beta|^{4}+32|\alpha \beta|^{2}+16|\alpha|^{4}+|\beta|^{6}-|\alpha|^{6}-3|\beta|^{4}|\alpha|^{2}+3|\beta|^{2}|\alpha|^{4}\right), \\
g_{\alpha \beta} & =\bar{\alpha} \bar{\beta}\left(-2048+256|\alpha|^{2}+256|\beta|^{2}-8|\alpha|^{4}+16|\alpha \beta|^{2}-8|\beta|^{4} .\right.
\end{aligned}
$$

In view of the structure of $C G C$ with first row equal to zero, the left rank reducing smoother

$$
M_{l}=F_{r 1}=\left(\begin{array}{cccc}
4 & -\alpha & -\beta & 0 \\
0 & 4 & 0 & -\beta \\
0 & 0 & 4 & -\alpha \\
0 & -\bar{\beta} & -\bar{\alpha} & 4
\end{array}\right)
$$

leads to $S_{l}=I_{4}-M_{l}^{-1} F=M_{l}^{-1}\left(M_{l}-F\right)$ with nonzeros only in the first column of $M_{l}-F$ and therefore symbol $T G S(x, y)=0$. So the Twogrid method requires only one iteration step.

If we switch to the full $n^{2} \times n^{2}$ matrices we use as prolongation the matrix relative to the product of the Block Toeplitz matrices

$$
B:=T_{n^{2}}(2-\cos (x)+\cos (y)) \cdot T_{n^{2}}(2+\cos (x)-\cos (y)) \cdot T_{n^{2}}(2+\cos (x)+\cos (y)),
$$

and as left smoother the matrix related to the above symbol $M_{l}$. Then for the full matrix with coarsening $\tilde{B}$ related to the index set $2: 2: n$ in both dimensions

$$
M_{l} \cdot T G S=\left(M_{l}-A\right)\left(I-\tilde{B}^{T} E^{T}\left(E \tilde{B} A \tilde{B}^{T} E^{T}\right)^{-1} E \tilde{B} \cdot A\right)
$$


TABLE 3.6

$T G S(x, y)=S_{l} \cdot C G C \cdot S_{r}$ for different smoothers (first row: norm, second row: spectral radius) for 9-point stencil. Again, * denotes the application of the same smoother as pre- and postsmoother.

\begin{tabular}{c||c|c|c|c|c|c|c} 
& $S_{J(8 / 9), *}$ & $S_{G S, *}$ & $S_{B G S, *}$ & $S_{\tilde{G}, *}$ & $S_{B \tilde{G} S, *}$ & $S_{F_{c 1}}, S_{F_{r 4}}$ & $S_{F_{r 12}}^{2}, S_{F_{c 43}}^{2}$ \\
\hline $\max _{x, y}\|T G S(x, y)\|_{2}$ & 0.32 & 0.048 & 0.10 & 0.048 & 0.026 & 0.019 & 0.022 \\
\hline $\max _{x, y} \rho(T G S(x, y))$ & 0.11 & 0.041 & 0.045 & 0.040 & 0.016 & 0.015 & 0.014
\end{tabular}

we get norm zero, and therefore this Twogrid method needs only one smoothing iteration and therefore can be considered as a direct solver. To obtain the overall solution for the linear system with given matrix $A$ it remains to solve one linear system with the smoother, e.g. by pcg, and the coarse grid problem of size $n^{2} / 4$.

Remark 3: Note, that the derived thick projection is interesting also for other problems. So in $[9,10]$ this projection is used efficiently for solving Helmholtz problems. Furthermore, in the following section we will derive also a more practical smoother for this thick projection applied on our 2D model problem. Hence, the proposed method is not as impractical as observed in [21, paragraph A.2.3].

3.3. Deriving Twogrid as a direct solver. In this section we will build the connection to the well known approach of Multigrid as a direct solver described in [21, paragraph A.2.3] by formulating these conditions in terms of the block symbol. We start by partitioning the given $k \times k$ block symbol $F$ and the vector $B_{1}=B(1,:)$ describing the projection, in the form

$$
\left(\begin{array}{cc}
F(1,1) & F(1,2: k) \\
F(2: k, 1) & F(2: k, 2: k)
\end{array}\right)=\left(\begin{array}{cc}
\gamma & g \\
g^{H} & G
\end{array}\right), B_{1}=\left(\begin{array}{ll}
B(1,1) & B(1,2: k)
\end{array}\right)=\left(\begin{array}{ll}
\delta & d
\end{array}\right) .
$$

The Galerkin coarse problem is then given by $B_{1} F B_{1}^{H}$. The goal in this approach is to obtain in the first row of the CGC symbol (1.1) a zero row. This leads to the condition

$$
\left(\begin{array}{ll}
\delta & d
\end{array}\right)\left(\begin{array}{cc}
\gamma & g \\
g^{H} & G
\end{array}\right)\left(\begin{array}{c}
\delta \\
d^{H}
\end{array}\right) \cdot I_{k}-\left(\begin{array}{c}
\delta \\
d^{H}
\end{array}\right)\left(\begin{array}{ll}
\delta & d
\end{array}\right)\left(\begin{array}{cc}
\gamma & g \\
g^{H} & G
\end{array}\right)
$$

has first row identical to zero. Considering the entries in columns $2: k$ yields

$$
\delta^{2} g+\delta d G=0 \Longrightarrow d=-\delta g G^{-1} .
$$

With this choice it turns out that also the first entry in the first row will be zero. In order to derive a sparse projection $B$ we can write $G^{-1}=G_{+} \operatorname{det}(G)$ and set $\delta=\operatorname{det}(G)$. Then $B$ is fully defined by its first row $B_{1}$ which is given by

$$
B_{1}=\left(\operatorname{det}(G)-g \cdot G_{+}\right)
$$

and this defines a sparse projection $B$ which can be described in terms of $G$ and the adjoint $G_{+}$by trigonometric polynomials. For the example from section 3.2 the resulting projection for the 5-point stencil is exactly the thick projection $\tilde{B}$ in (3.6).

By obtaining a zero column in the first row of the CGC block symbol it is obvious how we have to choose the smoother $M$ to derive $T G S \equiv 0$ :

$$
0=\left(I-M^{-1} F\right) \cdot C G C=M^{-1} \cdot(M-F) \cdot\left(\begin{array}{c}
0 \\
*
\end{array}\right),
$$


if $M$ coincides with $F$ up to the first column. Furthermore, $M$ has to be invertible. So we set

$$
M=\left(\begin{array}{cc}
F(1,1) & F(1,2: k) \\
0 & F(2: k, 2: k)
\end{array}\right)=\left(\begin{array}{cc}
\gamma & g \\
0 & G
\end{array}\right) .
$$

Disadvantages of this approach are that the projection and therefore also the coarse grid matrix might get thicker, and for the smoother we need to solve a linear system in $G=F(2: k, 2: k)$.

For $C G C$ symbol with first row zero we can also look for an approximate inverse smoother $N$ that yields $T G S \equiv 0$. It holds

$$
0=(I-N F) \cdot C G C=(I-N F) \cdot\left(\begin{array}{l}
0 \\
*
\end{array}\right) \Longrightarrow I-N F=\left(\begin{array}{ll}
* & 0
\end{array}\right),
$$

and therefore $N=F^{-1}-c \cdot e_{1}^{T} F^{-1}$ with some vector $c$.

Going back to the problem in section 3.2 the thick projection and the projection derived here by the approach from [21, paragraph A.2.3] formulated in block symbols are identical. But with the block symbol method we find as further approximate inverse solution $N$ with $T G S \equiv 0$ the block symbol

$$
N(x)=\left(\begin{array}{cccc}
0 & 0 & 0 & 0 \\
-\frac{1}{2 \alpha} & \frac{1}{8} & -\frac{\beta}{8 \alpha} & 0 \\
-\frac{1}{2 \beta} & -\frac{\alpha}{8 \beta} & \frac{1}{8} & 0 \\
-\frac{2}{\alpha \beta} & -\frac{1}{2 \beta} & -\frac{1}{2 \alpha} & 0
\end{array}\right) .
$$

In each smoothing step with $N(x)$ we have to multiply a vector with the related full matrix $N$; in view of the relation between block symbol and full matrix in colored ordering this product involves products with matrices e.g. related to the function $\alpha / \beta$. As in (3.5) the resulting block matrix can be written in the form $T(\alpha) \otimes$ $T(1 / \beta)=(T(\alpha) \otimes I) \cdot\left(I \otimes T(\beta)^{-1}\right)$. Hence, the multiplication with matrix $N$ can be done by simple products or solves with bidiagonal matrices related to $T(\alpha)$ and $T(\beta)$. This shows again the advantages of using approximate inverse smoothers. The condition $T G S \equiv 0$ can be satisfied by $M$ or $N$, but $N$ gives a more practical smoother based on bidiagonal solves while $M$ leads to a smoother that can be solved only iteratively. The disadvantage of the thicker coarse problem can be overcome e.g. by rediscretization in order to reduce the sparsity pattern of $A_{c}$. Then the algorithm will be no direct solver anymore, but is an overall practical modification of standard Multigrid methods. However, such a truncation of the Galerkin operator might lead to a diverging algorithm and can only be applied with great care. Following [21, paragraph A.2.4], in order to reduce the complexity of the coarse grid problem the truncation of the prolongation can be done without loss of convergence.

Until now we were studying the case that the projection was chosen in such a way that the rank deficient CGC symbol has first row equal zero. Much more interesting is the case that for given stencil of the original problem we have chosen our favorite projection and now we want to analyze the task whether it is possible to determine a practical smoother that leads to $T G S \equiv 0$. In the following we only consider the combination of left (post-smoother) and coarse grid correction in $S_{l} \cdot C G C \equiv 0$. To find a solution to this problem we need a factorization of the $k \times k$ symbol of rank $k-1$ of the form

$$
f_{c} \cdot C G C=\left(\begin{array}{lll}
d_{1} & \cdots & d_{k-1}
\end{array}\right)\left(\begin{array}{c}
c_{1} \\
\vdots \\
c_{k-1}
\end{array}\right)=D \cdot C,
$$


e.g. by choosing $k-1$ linearly independent rows or columns of $C G C$. Here, we have introduced the scalar factor $f_{c}$ representing the symbol of the Galerkin coarse matrix, in order to simplify the block symbol. By $H$ we denote the block symbol $F D$.

This leads to the conditions $0=(I-N F) D=D-N H$ or $0=(M-F) D=$ $M D-H$. Hence, we are looking for smoothers $N$ or $M$ satisfying the equations $D=N H$, resp. $M D=H$. We can partition the matrices $H, N, M$, and $D$ in the form

$$
H=\left(\begin{array}{c}
\tilde{h} \\
\tilde{H}
\end{array}\right), \quad N=\left(\begin{array}{ll}
\tilde{n} & \tilde{N}
\end{array}\right), \quad M=\left(\begin{array}{ll}
\tilde{m} & \tilde{M}
\end{array}\right), \quad D=\left(\begin{array}{c}
\tilde{d} \\
\tilde{D}
\end{array}\right)
$$

with square submatrices $\tilde{H}, \tilde{N}, \tilde{M}$, and $\tilde{D}$, and vectors $\tilde{h}, \tilde{n} \tilde{m}$, and $\tilde{d}$. This leads to the solutions

$$
N=\left(\begin{array}{ll}
0 & D \tilde{H}^{-1}
\end{array}\right)+\tilde{n} \cdot\left(\begin{array}{ll}
1 & -\tilde{h} \tilde{H}^{-1}
\end{array}\right), M=\left(\begin{array}{ll}
0 & H \tilde{D}^{-1}
\end{array}\right)+\tilde{m} \cdot\left(\begin{array}{ll}
1 & -\tilde{d} \tilde{D}^{-1}
\end{array}\right) .
$$

So in this form $N$ and $M$ are given in terms of the block symbol $C G C$ and for each choice of vector $\tilde{n}$ or $\tilde{m}$ in $\mathbf{R}^{k-1}$ we get a smoother satisfying $T G S \equiv 0$. The remaining task is to identify vectors $\tilde{n}$, resp. $\tilde{m}$, that give practical smoothers $N$ and $M$, e.g. smoothers that can be described by trigonometric polynomials (to enforce sparsity) and - in the case of $M$ - lead to a linear system that is easy to solve. To this aim we have to look for a $k-1 \times k-1$ submatrix of $D$ or $H$ that is invertible with simple determinant. We have to postpone this project to future work.

4. Conclusions. By transforming all appearing matrices in terms of generating block symbols we derive a compact Fourier analysis for Multigrid methods where the block symbols capture the behavior of the Multigrid solver sufficiently. This approach can also be applied to general constant coefficient PDEs, e.g. for anisotropic equations or the Helmholtz problem $([9,10])$. Furthermore, the derived projections and smoothers can also be applied for the general case of varying coefficients. The new approach leads to a generalization of the results in [21, paragraph A.2.3] on Multigrid as a direct solver, and allows to derive and analyze approximate inverse smoothers, rank reducing smoothers, and generalized projections. The compact Fourier analysis is helpful for analyzing the smoothing behavior and the total Twogrid error reduction.

In the future this approach will be applied on the aggregation Multigrid method and on more general PDEs. A Three-grid and Four-grid analysis shall be derived. Furthermore, the block symbol can be used to develop a software tool for symbolic and numerical computations to derive and analyze Multigrid methods. 


\section{REFERENCES}

[1] A. Arico, M. Donatelli, And S. Serra, V-cycle optimal convergence of certain (multilevel) structured linear systems, SIAM J. Matrix Anal. Appl. Vol. 26 (1) (2004), p. 186-214.

[2] A. BRandt, Multi-level adaptive solutions to boundary value problems, Math. Comput. 31 (1977), p. 333-390.

[3] W.L. Briggs, V.E. Henson, and S.F. McCormick, A Multigrid tutorial, SIAM, Philadelphia, 2000.

[4] R. Chan, Q. Chang, and H. Sun, Multigrid methods for ill-conditioned symmetric Toeplitz Systems, SIAM J. Sci. Comput. 19 (1998), p. 516-529.

[5] T. Chan And H. Elman, Fourier Analysis of iterative methods for elliptic problems, SIAM Review 31 (1) (1989), p. 20-49.

[6] P.J. Davis, Circulant matrices, Wiley, New York, 1979.

[7] G. Fiorentino, and S. Serra, Multigrid methods for Toeplitz matrices, Calcolo Vol. 28 3-4 (1991), p. 283-305.

[8] G. Fiorentino and S. Serra, Multigrid methods for symmetric positive definite Block Toeplitz matrices with nonnegative generating functions, SIAM J. Sci. Comp. 17 (5) (1996), p. 10681081.

[9] R. Fischer, Multigrid methods for anisotropic and indefinite structured linear systems of equations, Ph.D. Thesis, TU München, 2006.

[10] R. Fischer And T. HuCKLe, Multigrid Methods for anisotropic BTTB Systems, Lin. Alg. Appl. Vol. 17 (2) (2006), p. 314-334.

[11] U. Grenander and G. Szegö, Toeplitz forms and their applications, Chelsea Publishing, 2nd edition, New York, 1984.

[12] T. Huckle and D. Noutsos, Preconditioning Block Toeplitz matrices, to appear in ETNA.

[13] T. Huckle and J. Staudacher, Multigrid Preconditioning and Toeplitz Matrices, ETNA Vol. 13 (2002), p. 81-105.

[14] T. Huckle and J. Staudacher, Multigrid methods for Block Toeplitz Matrices with small size blocks, BIT 46 (2006), p. 61-83.

[15] Y. Notay and P.S. Vassilevski, Recursive Krylov-based multigrid cycles, to appear in Numer. Lin. Alg. Appl..

[16] Y. Notay And A.C. Muresan, Analysis of aggregation-based multigrd, to appear in SIAM J. Sci. Comp..

[17] J. Ruge and K. Stüben, Algebraic Multigrid, in Multigrid Methods, ed. by S. Mccormick, SIAM, Philadelphia, pp. 73-130, 1987.

[18] S. Serra, Optimal, quasioptimal and superlinear band-Toeplitz preconditioners for asymptotically ill-conditioned positive definite Toeplitz systems, Math. Comp. 66 (218) (1997), p. 651-665.

[19] S. SERRA, Spectral and computational analysis of Block Toeplitz matrices having nonnegative definite matrix-valued generating functions, BIT 39(1) (1999), p. 152-175.

[20] S. Serra And C. Tablino Possio, Multigrid methods for multilevel circulant matrices, SIAM J. Sci. Comp. Vol. 26 (1) (2004), p. 55-85.

[21] U. Trottenberg, C.W. Osterlee, And A. Schüller, Multigrid, Acad. Press., San Diego, 2001.

[22] H. Widom, Asymptotic behavior of block Toeplitz matrices and determinants II, Adv. Math. 21 (1976), p. 1-29.

[23] R. Wienands and W. Joppich, Practical Fourier analysis for multigrid methods, Chapman \& Hall/CRC, Boca Raton, 2005. 